\title{
Transcription elongation regulator 1 (TCERG1) regulates competent RNA polymerase II-mediated elongation of HIV-1 transcription and facilitates efficient viral replication
}

\author{
Mayte Coiras $^{1 \dagger}$, Marta Montes ${ }^{2,5 \dagger}$, Immaculada Montanuy ${ }^{2,6}$, María Rosa López-Huertas ${ }^{1}$, Elena Mateos ${ }^{1}$, \\ Caroline Le Sommer ${ }^{3,7}$, Mariano A Garcia-Blanco ${ }^{3}$, Cristina Hernández-Munain ${ }^{4}$, José Alcamí and Carlos Suñé ${ }^{*}$
}

\begin{abstract}
Background: Control of RNA polymerase II (RNAPII) release from pausing has been proposed as a checkpoint mechanism to ensure optimal RNAPII activity, especially in large, highly regulated genes. HIV-1 gene expression is highly regulated at the level of elongation, which includes transcriptional pausing that is mediated by both viral and cellular factors. Here, we present evidence for a specific role of the elongation-related factor TCERG1 in regulating the extent of HIV-1 elongation and viral replication in vivo.

Results: We show that TCERG1 depletion diminishes the basal and viral Tat-activated transcription from the HIV-1 LTR. In support of a role for an elongation mechanism in the transcriptional control of HIV-1, we found that TCERG1 modifies the levels of pre-mRNAs generated at distal regions of HIV-1. Most importantly, TCERG1 directly affects the elongation rate of RNAPII transcription in vivo. Furthermore, our data demonstrate that TCERG1 regulates HIV-1 transcription by increasing the rate of RNAPII elongation through the phosphorylation of serine 2 within the carboxyl-terminal domain (CTD) of RNAPII and suggest a mechanism for the involvement of TCERG1 in relieving pausing. Finally, we show that TCERG1 is required for HIV-1 replication.

Conclusions: Our study reveals that TCERG1 regulates HIV-1 transcriptional elongation by increasing the elongation rate of RNAPII and phosphorylation of Ser 2 within the CTD. Based on our data, we propose a general mechanism for TCERG1 acting on genes that are regulated at the level of elongation by increasing the rate of RNAPII transcription through the phosphorylation of Ser2. In the case of HIV-1, our evidence provides the basis for further investigation of TCERG1 as a potential therapeutic target for the inhibition of HIV-1 replication
\end{abstract}

Keywords: TCERG1, Transcription elongation, RNA polymerase II, Pausing

\section{Background}

Gene transcription begins with the recruitment and assembly of an RNA polymerase II (RNAPII) complex on core promoter sequences, followed by initiation of RNA synthesis and subsequent elongation. Most of the initial studies to improve the understanding of transcriptional regulation were focused on the initiation stage and the mechanism by which the RNAPII complexes were

\footnotetext{
* Correspondence: csune@ipb.csic.es

${ }^{\dagger}$ Equal contributors

${ }^{2}$ Department of Molecular Biology, Instituto de Parasitología y Biomedicina

"López Neyra" (IPBLN-CSIC), Armilla, Granada 18016, Spain

Full list of author information is available at the end of the article
}

recruited and assembled on promoters (reviewed in [1]). However, transcription elongation is also a major target for gene regulation. Initial studies of the $H s p 70$ gene in Drosophila have shown that RNAPII complexes stall in the $5^{\prime}$ region of the transcription unit [2]. Paused RNAPII within the promoter-proximal region has also been observed at many proto-oncogenes, such as $c-m y b$ [3], $c$-mos [4], c-myc [5], and c-fos [6], and other mammalian genes [7-9], suggesting that RNAPII pausing at promoterproximal regions is predominantly the rule rather than the exception. More recently, genome-wide results from

\section{Biomed Central}

(C) 2013 Coiras et al.; licensee BioMed Central Ltd. This is an open access article distributed under the terms of the Creative Commons Attribution License (http://creativecommons.org/licenses/by/2.0), which permits unrestricted use, distribution, and reproduction in any medium, provided the original work is properly cited. 
humans and Drosophila have provided strong evidence for the widespread existence of paused RNAPII at gene promoter-proximal regions [10-15]. Thus, increasing evidence suggests that RNAPII often arrests within the vicinities of the start site of transcription and that release of paused RNAPII might act as an important checkpoint to ensure optimal RNAPII activity, especially in large, highly regulated genes (reviewed in [16]). In addition to promoter-proximal pausing, two recent reports using global analysis of nascent RNA in yeast have shown that RNAPII transiently accumulates at specific sites before termination and around 3' splice sites of genes $[17,18]$. Although the nature and position of the observed pausing differ between the studies, a strong correlation between paused RNAPII and cotranscriptional splicing was observed. These observations agree with the model in which transcriptional pausing acts as a regulatory checkpoint, in this case for a downstream processing event.

Differences in the phosphorylation status of the tandem repeats of the consensus heptapeptide YSPTSPS in the carboxyl-terminal domain (CTD) of the largest subunit of RNAPII have been associated with the location of RNAPII on the gene. Hypophosphorylated RNAPII assembles into pre-initiation complexes at gene promoters, whereas hyperphosphorylated RNAPII associates with the elongation stage. Cyclin-dependent kinase 7 (CDK7), which is part of the general transcription factor IIH (TFIH), is responsible for the phosphorylation of serines at the fifth position (Ser5). This modification has been linked with transcription initiation and is preferentially associated with the 5 '-end of the genes. As RNAPII elongates further downstream, phosphorylation of serines at the second position (Ser2) increases, which is viewed as an elongation mark and is preferentially associated with the 3 '-end of the genes. The transition between Ser 5 and Ser 2 phosphorylation is essential for the release of paused RNAPII complexes from the proximal promoter and represents an important mechanism of control for proper elongation. Extensive phosphorylation of Ser5 has also been found in the CTD of RNAPII paused around 3' splice sites of genes [17]. Phosphorylation of Ser2 of the CTD could conceivably also be associated with release from this pausing. Although the correlation between Ser2 phosphorylation and elongation is widely accepted, recent discoveries related to modifications of the CTD and the enzymes involved show that this model is an oversimplified representation of a more complex process regulating elongation control of gene expression (reviewed in [19-21]).

Human Immunodeficiency virus type 1 (HIV-1), the etiologic agent of acquired immunodeficiency syndrome (AIDS), is a complex lentivirus with a highly regulated life cycle. Upon infection, HIV-1 integrates into the host genome DNA where it remains in a silent state, which is critical in latently infected cells. The chromatin structure, as well as a specific group of proteins, plays a role in repressing viral gene expression by causing the RNAPII complexes to pause in the $5^{\prime}$ region of the transcription unit. It is now well established that Nuc-1, a nucleosome located immediately downstream of the HIV-1 transcriptional initiation site, directly represses promoter activity and is mainly responsible for transcriptional silencing in latently infected cells. Consequently, the synthesized transcripts fail to support viral replication [22]. In recent work, a mechanism involving the microprocessor complex as well as specific termination factors has been proposed to regulate pausing and premature termination at the HIV-1 Long Terminal Repeat (LTR) [23]. HIV-1 transcription is controlled by cis-acting sequences located at the $5^{\prime}$ LTR and by both viral and cellular trans-acting factors [24]. Among other sequences, the LTR contains a canonical TATA-box sequence followed by two NF- $\mathrm{kB}$ and three Sp1 sites that constitute the core promoter and modulate basal and activated viral transcription [25]. Activation of this promoter requires expression of the viral regulatory protein Tat, which binds to a 59-nt stem-bulge-loop structured RNA element named TAR (for trans-activation-responsive region) located at the 5 '-end of all HIV-1 nascent transcripts [26]. The transcriptional trans-activation of the HIV-1 promoter by Tat requires specific cellular cofactors. The complex $\mathrm{P}-\mathrm{TEFb}$ is a Tat cofactor that is essential for the transcriptional function of Tat [27-29] and is recruited to the TAR element through the interaction of Tat with cyclin $\mathrm{T} 1$ (a component of P-TEFb). The CDK9 component of PTEFb phosphorylates Ser2 within the CTD of RNAPII as well as several positive and negative elongation factors. These events lead to release of the arrested RNA complexes in the $5^{\prime}$ region of the transcription unit, resulting in a potent stimulation of the overall rate of transcriptional elongation and productive HIV-1 replication. In addition to the essential role of the Tat/TAR axis in the control of HIV-1 gene expression and latency, the HIV TATA-box and immediately flanking sequences have been shown to be specifically required for the production of specific pre-initiation complexes that are targets of trans-activation by Tat [30-35]. In this context, Tat plays a highly specific role in the recruitment of transcription factors and co-activators through its multifaceted interactions with different cellular components [36-44]. Therefore, HIV-1 transcription is a critical step in the viral life cycle, and the intricate regulation of this transcription provides the basis for understanding viral transcriptional latency [45-48].

In addition to P-TEFb, other factors have been implicated in the mechanism of Tat-mediated transcriptional activation. Among these, TCERG1 (HUGO approved gene 
name: transcription elongation regulator 1; previously designated CA150, for co-activator of $150 \mathrm{kDa}$ ) is a nuclear protein that was first detected in a fraction from a wild-type Tat affinity column that contained an activity required for transcription activation by Tat in an in vitro transcription system [49]. We have previously shown that immunodepletion of TCERG1 from nuclear extracts decreases Tat activation of RNAPII elongation efficiency in vitro, suggesting that TCERG1 might play a positive role in this process [49]. We have also shown that transient overexpression of TCERG1 protein in HEK293T cells reduces the activity of HIV-1 basal and Tat-activated transcription of the HIV-1 LTR by decreasing the level of elongation-competent transcription complexes. We hypothesized that overexpression of TCERG1 might alter the composition of the Tat-responsive transcription complexes, e.g. by sequestering a limiting Tat-cofactor [50]. Based on these and other data, such as the interaction of TCERG1 with several components of the elongation machinery [51] and with the phosphorylated CTD of RNAPII [52], TCERG1 appears to function in the elongation stage of transcription. The aforementioned data strongly suggested that TCERG1 is a cofactor for HIV-1 transcriptional elongation and thus might be a relevant protein in the HIV-1 life cycle. However, this hypothesis has not previously been formally tested in vivo.

Very recently, we have demonstrated that TCERG1 regulates the expression of the apoptosis gene $B c l-x$ by modulating the rate of RNAPII transcription. In this previous work, we proposed that TCERG1 acts on RNAPII to relieve pausing, thus acting as a checkpoint factor to ensure optimal RNAPII activity during elongation [53]. These results posit the question of whether the effect of TCERG1 on $\mathrm{Bcl}-x$ elongation is a more general mechanism that operates in other genes that harbor pause sites, e.g., in HIV-1. In this manuscript, we evaluate the role of endogenous TCERG1 in HIV-1 transcription and viral replication in vivo. We found that TCERG1 depletion diminishes the basal and Tat-activated transcription from the HIV-1 LTR by impairing elongation of the HIV-1 transcripts. Most importantly, TCERG1 affects the rate of RNAPII transcription of HIV-1. We also observed that the TCERG1-mediated transcriptional effect is associated with changes in the pattern of Ser2 phosphorylation in the RNAPII CTD. In addition, we show that TCERG1 is required for HIV-1 replication, as depletion of TCERG1 decreased viral replication in Jurkat cells and peripheral blood lymphocytes (PBLs). Based on our data, we propose a general mechanism for TCERG1 acting on genes that are regulated at the level of elongation by increasing the rate of RNAPII transcription through the phosphorylation of Ser2. In the case of HIV-1, our evidence provides the basis for further investigation of
TCERG1 as a potential therapeutic target for the inhibition of HIV-1 replication.

\section{Results}

\section{TCERG1 depletion decreases basal and Tat-activated} transcription from the HIV-1 LTR

As a first approach to test the role of TCERG1 in HIV-1 transcription, we examined PBLs co-transfected with a luciferase expression vector under the control of the HIV-1 LTR promoter along with the following vectors to induce TCERG1 mRNA interference: pGeneClip-shTCER G1-C1 or pGeneClip-shTCERG1-3 and pGeneClip-sh TCERG1-4, and the latter pair together with pGeneClipshTCERG1-1. TCERG1 knockdown was assessed by quantitative RT-PCR assay (qRT-PCR) (Figure 1A). PBLs were transfected in a resting state and then maintained with IL-2 for 48 hours. Upon quantification of the luciferase activity, we observed that TCERG1 knockdown significantly reduced the basal transcription of the LTR (Figure 1A, left panel). Similar results were observed when the experiment was performed in the presence of Tat. Tat-mediated transcriptional activation of the luciferase reporter gene was reduced upon TCERG1 depletion in PBLs (Figure 1A, right panel). Tat expression in the nucleus of the transfected cells was assessed by immunofluorescence (Figure 1A).

To ensure long-term, reproducible, and defined silencing effects, we generated Jurkat cells with stable TCERG1 mRNA interference. Jurkat cell lines were constructed by transfection of pGeneClip-shTCERG1 vectors. Jurkat-shTCERG1-(3-4) and Jurkat-shTCERG1(1-3-4) cell lines were generated by co-transfection with pGeneClip-shTCERG1-3 and pGeneClip-shTCERG1-4 and/or pGeneClip-shTCERG1-1 vectors. The control Jurkat-shTCERG1-C1 cell line was generated by transfection with the pGeneClip-shTCERG1-1 vector. All of the cell lines were tested for shRNA-mediated TCERG1 depletion by semiquantitative RT-PCR assay. JurkatshTCERG1-(3-4) and Jurkat-shTCERG1-(1-3-4) cell lines showed approximately $70 \%$ and $80 \%$ TCERG1 depletion, respectively (Figure $1 \mathrm{~B}$ ). In agreement with our previous data, we observed significantly reduced basal and Tatactivated transcription from the HIV-1 LTR upon TCERG1 knockdown in these Jurkat cells (Figure 1B). We noted that the inhibition of transcription was reduced in the presence of Tat in both cell systems (compare right and left panels in Figure 1). This result might indicate that TCERG1 acts on basal transcription rather that on Tat-mediated trans-activation. Immunofluorescence analysis indicated that TCERG1 has no effect on the spatial localization of Tat (Figure 1B). Together, these findings indicate that TCERG1 depletion affects basal and Tat-mediated transcriptional activation of the HIV-1 LTR in lymphoid cells. We conclude that 


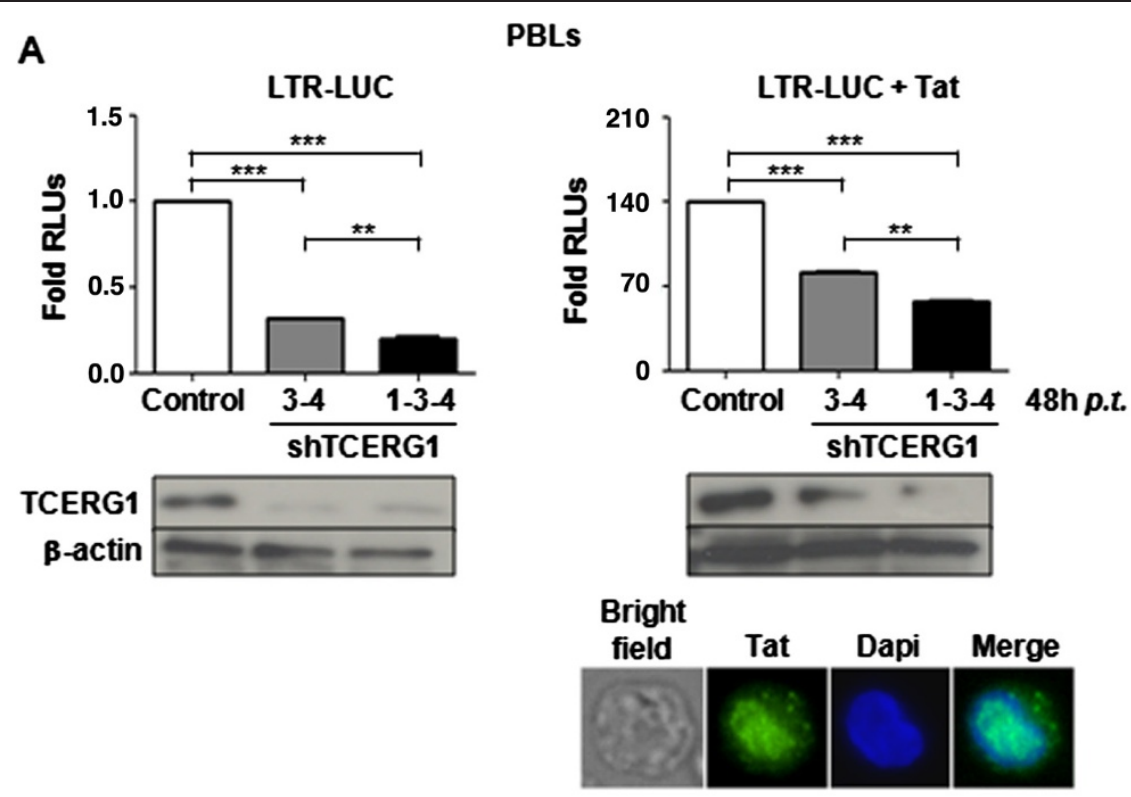

B

Jurkat shTCERG1
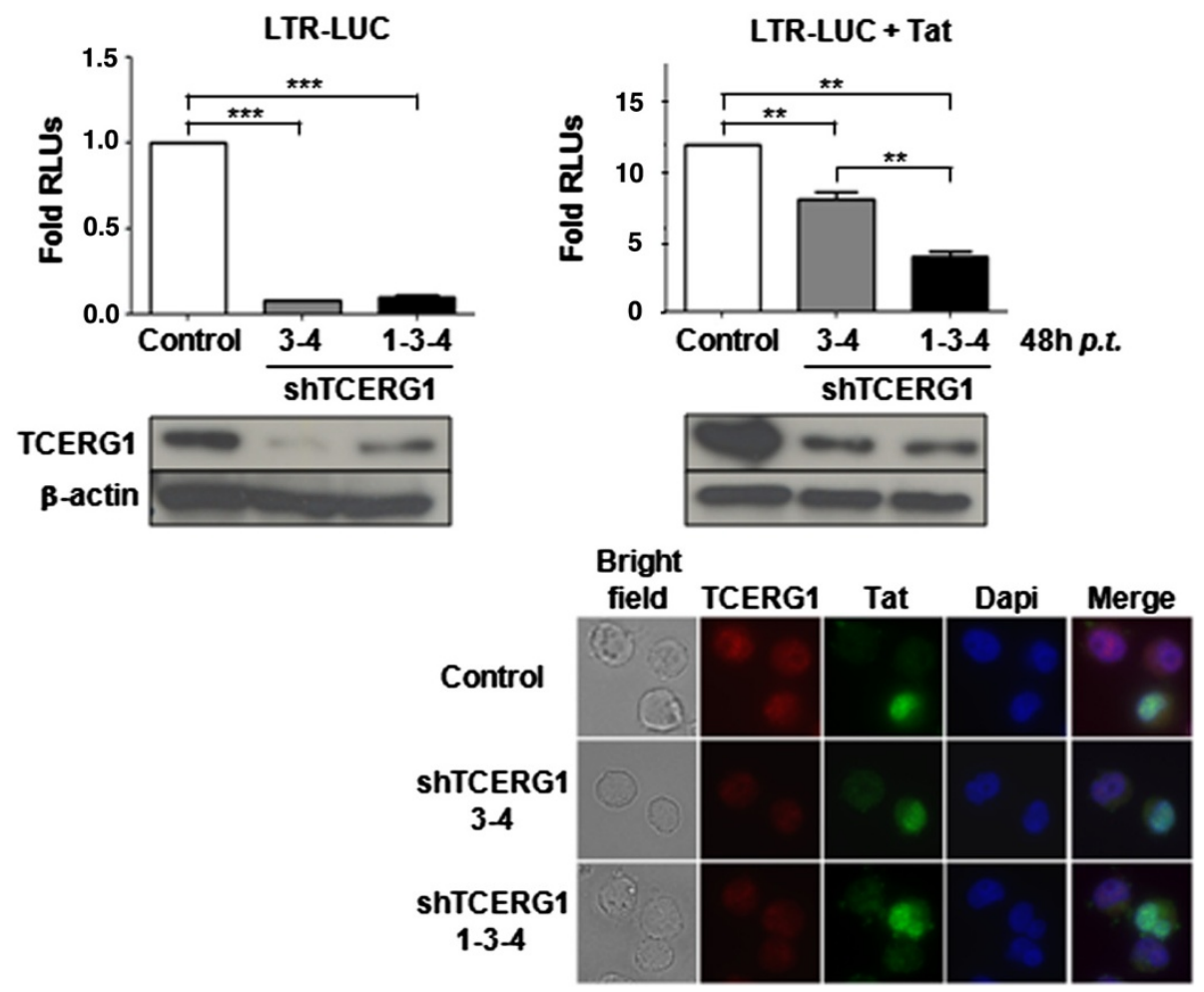

Figure 1 (See legend on next page.) 
(See figure on previous page.)

Figure 1 TCERG1 depletion decreases basal and Tat-activated transcription from the HIV-1 LTR. A. PBLS were co-transfected with a luciferase expression vector under the control of the HIV-1 LTR promoter (LTR-LUC) along with pGeneClip-shTCERG1-C1 or pGeneClip-shTCERG1-3 and pGeneClip-shTCERG1-4, and the latter pair together with pGeneClip-shTCERG1-1. The luciferase activity was quantified 72 hours after transfection as relative light units (RLUs) using the control cells as the basal signal. Data from three independent experiments are represented in the histogram (means \pm SEM). Statistical analysis was performed and $p$-values are shown $\left.{ }^{* *}, p<0.01 ;^{* * *}, p<0.001\right)$. TCERG1 interference was determined by immunoblotting. Appropriate expression and nuclear localization of Tat was evaluated by immunofluorescence using a specific antibody against Tat and Dapi to stain the nucleus (a representative cell is shown). B. Jurkat cells with stable interference of TCERG1 were co-transfected with a luciferase expression vector under the control of the HIV-1 LTR promoter (LTR-LUC) along with CMV-Tat101 or pCDNA3 as negative control. The luciferase activity was quantified 72 hours after transfection as relative light units (RLUs) using the control cells as the basal signal. The results of three different experiments are represented in the histogram (means \pm SEM). Statistical analysis was performed, and $p$-values are shown $\left({ }^{* *}, p<0.01 ;{ }^{* * *}, p<0.001\right)$. TCERG1 interference was determined by immunoblotting. The effect of TCERG1 interference on the expression and nuclear localization of Tat was analyzed by immunofluorescence in all Jurkat cell lines with stable interference of TCERG1.

TCERG1 plays a positive role in efficient transcription from the HIV-1 LTR.

\section{TCERG1 depletion impairs elongation of HIV-1 transcripts} To test whether TCERG1 affects HIV-1 transcriptional elongation, we measured the amount of transcripts generated at proximal and distal regions of the HIV-1 gene. We transfected the Jurkat cell lines with the pNL4-3-wt viral clone and assessed the amount of viral transcripts by qRT-PCR. We designed specific primers directed against the R/U5-gag and env/nef regions (Figure 2A). We found that both early and late elongation of viral transcripts was inhibited by approximately $50 \%$ in the Jurkat-shTCERG1 cell lines (Figure 2B, left panel). To lend support to the observation that TCERG1 affects elongation, we measured the pre-mRNAs generated at distal regions from a transiently transfected plasmid that contains the complete pro-virus genome with a deletion in the retrotranscriptase protein (pNL4-3 $\Delta \mathrm{RT}$ ) under conditions of TCERG1 knockdown in HEK293T cells. We performed these experiments in HEK293T cells because we have previously observed that TCERG1 affects the level of elongation-competent transcription complexes in these cells [50]. The pNL4-3 $\Delta$ RT DNA generates viral particles in HEK293T cells that are able to enter target cells, but due to the absence of reverse transcriptase, no replication is detected. The cDNA was synthesized using random hexamers followed by qPCR with primers amplifying a region corresponding to the env gene (Figure 2A). A plasmid expressing human growth hormone (pXGH5) was also transfected and amplified by qPCR as a transfection control (data not shown). The levels of RNA were normalized to those of endogenous GAPDH. Similarly with the data obtained in Jurkat cells (Figure 2B), we observed a diminished accumulation of distal transcripts by approximately 30-40\% upon TCERG1 knockdown (Figure 2C, left panel). The level of TCERG1 expression in silenced cells compared to that of CDK9 is also shown (Figure $2 \mathrm{C}$, right panel). This result confirms the previous data and further suggests that TCERG1 acts through an elongation mechanism in the regulation of HIV-1 gene expression.

To directly test whether TCERG1 affects the rate of RNAPII transcription, we used Padgett's protocol. In this procedure, 5,6-Dichlorobenzimidazole $1-\beta-D$-ribofuranoside (DRB), which reversibly blocks gene transcription in vivo by inhibiting the P-TEFb-dependent Ser 2 phosphorylation of the CTD of RNAPII, is used in combination with qRT-PCR to analyze the transcription of human genes [54]. To perform this protocol under optimal conditions, we used T-Rex-HEK293 cell lines, which ensure high transfection efficiency, in which expression of control shRNA and shRNA targeting TCERG1 can be induced by addition of tetracycline. The analysis of cell lysates from induced cells showed that the cells that contain shRNAs targeting TCERG1 express significantly lower TCERG1 protein levels compared to the control shRNAs (Figure 2D, right panel). Control and TCERG1knockdown cells were transfected with pNL4-3 $\triangle \mathrm{RT}$ and pXGH5. The cells were treated with DRB, and samples were collected at different time points after DRB removal. Quantitative RT-PCR was performed using the primers indicated above to amplify a region corresponding to the env gene (Figure 2A). The DRB-treated control cells were able to recover transcription within 20 to $80 \mathrm{~min}$ after DRB removal, which is consistent with a transcriptional lag due to the genomic distance from the start site of transcription [54]. In contrast, transcriptional recovery in TCERG1-knockdown cells was significantly slower (Figure 2D, left panel). These results demonstrate a role for TCERG1 in the RNAPII transcription of HIV-1 in vivo.

\section{TCERG1 is present at the promoter and coding regions of the HIV-1 gene}

The above functional results and other published data $[51,53,55,56]$ indicate that TCERG1 associates with components of the transcriptional machinery to regulate HIV1 transcriptional elongation. The point at which TCERG1 associates with the transcriptional complex during HIV-1 transcriptional activation remains an important question in understanding TCERG1 function. To address this issue, 


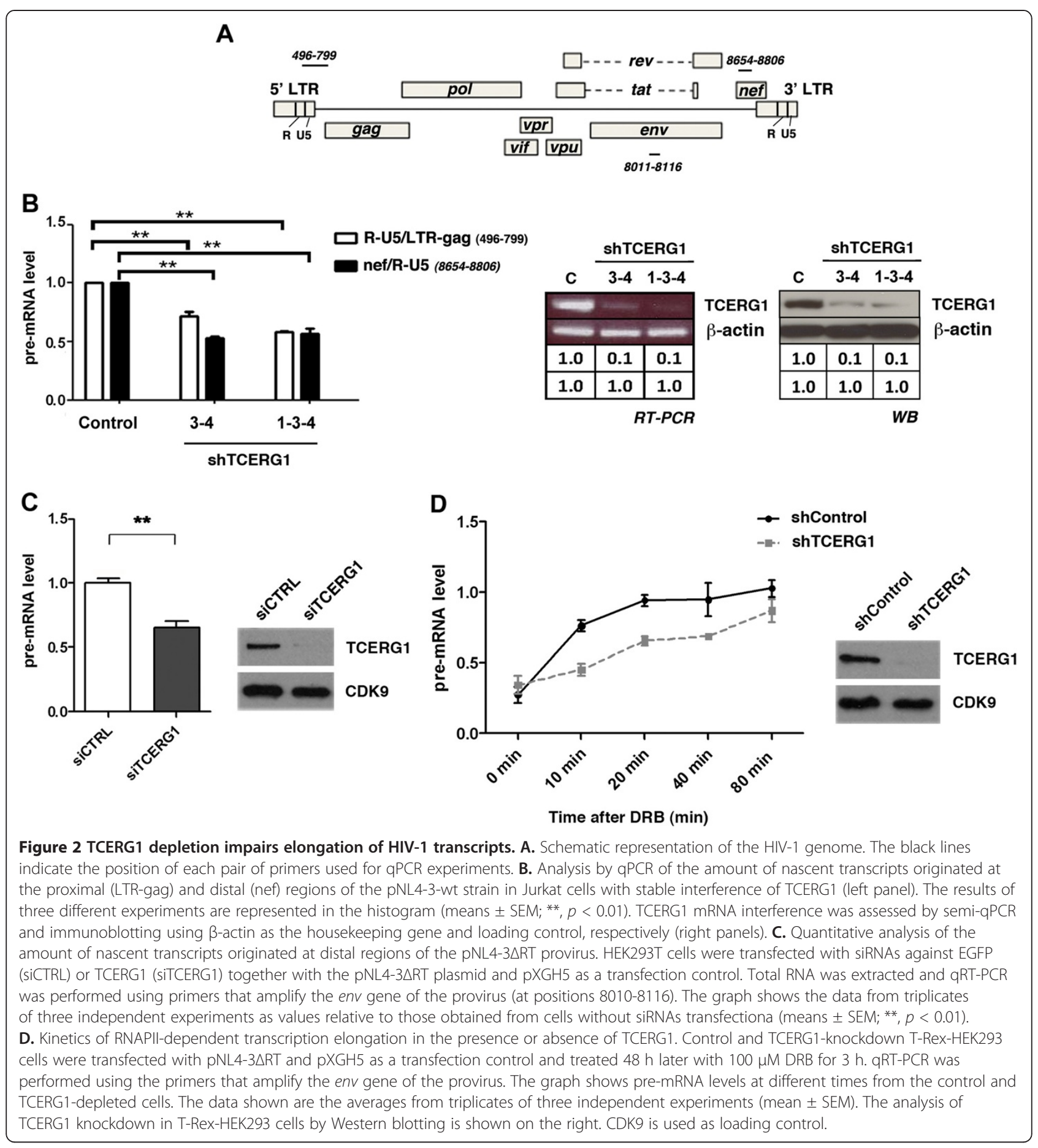

we used a combination of in vitro and in vivo approaches. First, to directly measure the transcribing RNAPII in an in vitro transcription assay, we used an immobilized template consisting of a biotinylated double G-less cassette template driven by the HIV-1 LTR to isolate HIV-1 RNAPII pre-initiation complexes (PICs) [35]. This template synthesizes transcripts that contain two regions (cassettes) of different sizes that lack guanosine residues; therefore, these G-less cassettes are resistant to digestion with RNase T1. One G-less cassette is located proximal to the promoter, enabling measurement of the numbers of transcription complexes that reach nucleotide +183 (short), while the second downstream G-less cassette measures the number of transcripts beyond nucleotide +1960 (long) (see Figure 3A). The biotinylated templates were incubated with HeLa nuclear extract and subsequently isolated 
A

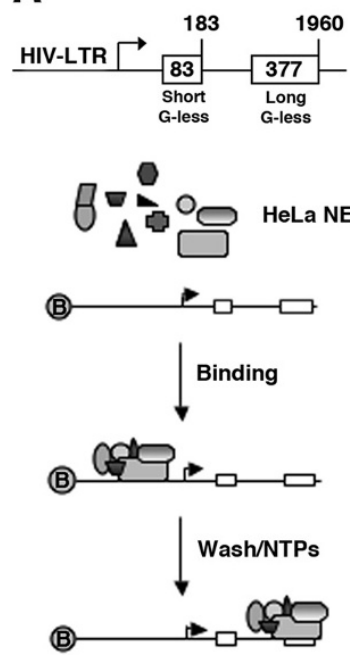

B

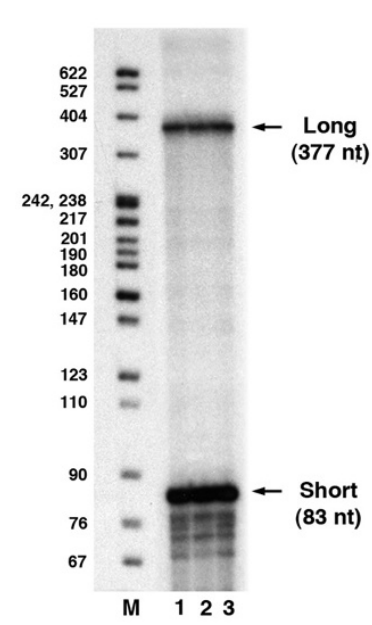

C

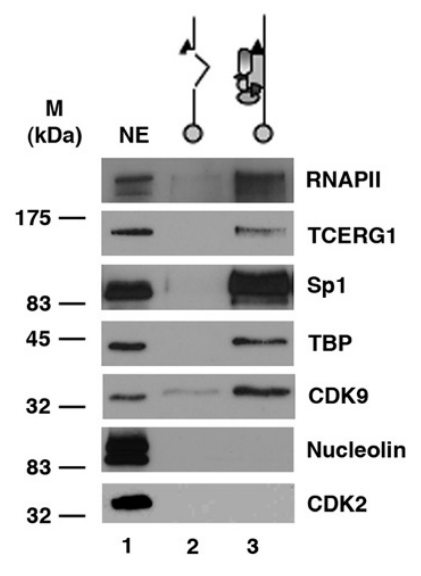

Figure 3 Analysis of PICs assembled on the HIV-1 promoter in vitro. A. Experimental strategy for analyzing active PICs. Step 1: Cell-free transcription reactions are performed using HeLa nuclear Extract (NE) and biotinylated templates carrying the HIV-1 LTR. Step 2: Transcription complexes are purified by binding to streptavidin-coated magnetic beads. Step 3: Nascent RNA chains are labeled by incorporation of a-[32P]UTP. A schematic representation of the HIV-1 double G-less cassette template used in the experiment is shown at the top of the panel. B. In vitro transcription reactions were performed with the purified PICs. Lanes 1-3 represent three independent reactions performed using the HIV-1 template. Arrows indicate the migration of long and short transcripts. C. TCERG1 is a component of the HIV-1 PICs. PICs were formed on constructs containing (lane 3) or not (lane 2) the HIV-1 promoter. PICs were purified with streptavidin-coated magnetic beads, and Western blot analysis of the purified PICs was performed using the specific antibodies shown at the right side of the panel. The relative mobilities (in kDa) of the molecular mass markers $(M)$ are shown on the left side of the panel.

using streptavidin-coated magnetic beads. We performed in vitro transcription assays to test the activity of the isolated transcription complexes. We observed clear signals for the short and long transcripts (Figure 3B), which indicated that the isolated complexes were competent to transcribe the DNA sequences. We next analyzed the relative protein composition of the PICs formed on the HIV-1 promoter sequences, especially with respect to TCERG1. Figure $3 \mathrm{C}$ shows a representative immunoblot analysis of PICs using RNAPII-, TCERG1-, Sp1-, TBP-, CDK9-, nucleolin-, and CDK2specific antibodies. As expected, RNAPII, Sp1, TBP, and CDK9 were detected in the PICs formed on the HIV-1 promoter. Interestingly, TCERG1 was also found in the PICs. We did not detect binding of nucleolin or CDK2 to the PICs. These results demonstrate the presence of TCERG1 in the RNAPII complexes assembled onto the HIV-1 promoter in vitro.

Next, we determined whether transiently transfected TCERG1 is recruited to the HIV-1 LTR in vivo by measuring the density of TCERG1 at specific regions of the pNL4-3 $\Delta$ RT gene using quantitative ChIP (qChIP) analysis. We designed specific primers to detect TCERG1 recruitment at the 5' LTR, gag, vif, env, and 3' LTR regions of the HIV-1 gene (Figure 4A). The qChIP experiments revealed a specific association of TCERG1 at all these regions (Figure 4B). TCERG1 was not detected using control IgG antibodies or control intergenic regions (Figure 4 and data not shown). These results indicate that TCERG1 specifically associates with both, the promoter and downstream regions of the HIV-1 gene in vivo.

\section{TCERG1 depletion decreases phosphorylation of Ser2} within the RNAPII CTD

The above results demonstrate a specific association of TCERG1 along the HIV-1 genome that resembles the pattern of the RNAPII recruitment onto a gene unit. In addition, transcriptional elongation is known to be dependent on RNAPII CTD phosphorylation along the transcribed gene $[57,58]$. Because TCERG1 has previously been shown to bind to the phosphorylated CTD of RNAPII [52], we examined whether the observed decrease in the elongation rate of the enzyme (Figure 2D) was due to changes in the CTD phosphorylation pattern. To perform these experiments, we analyzed the phosphorylation of the RNAPII CTD under conditions of TCERG1 knockdown or overexpression. We measured the density of RNAPII at the 5' LTR, gag, vif, env, and 3' LTR regions of a transiently transfected pNL4-3 $\Delta \mathrm{RT}$ provirus (see Figure 4A) by qChIP analysis using specific antibodies against total RNAPII (N20), Ser2, and Ser5 (Figure 5). TCERG1 depletion resulted in a decreased accumulation 

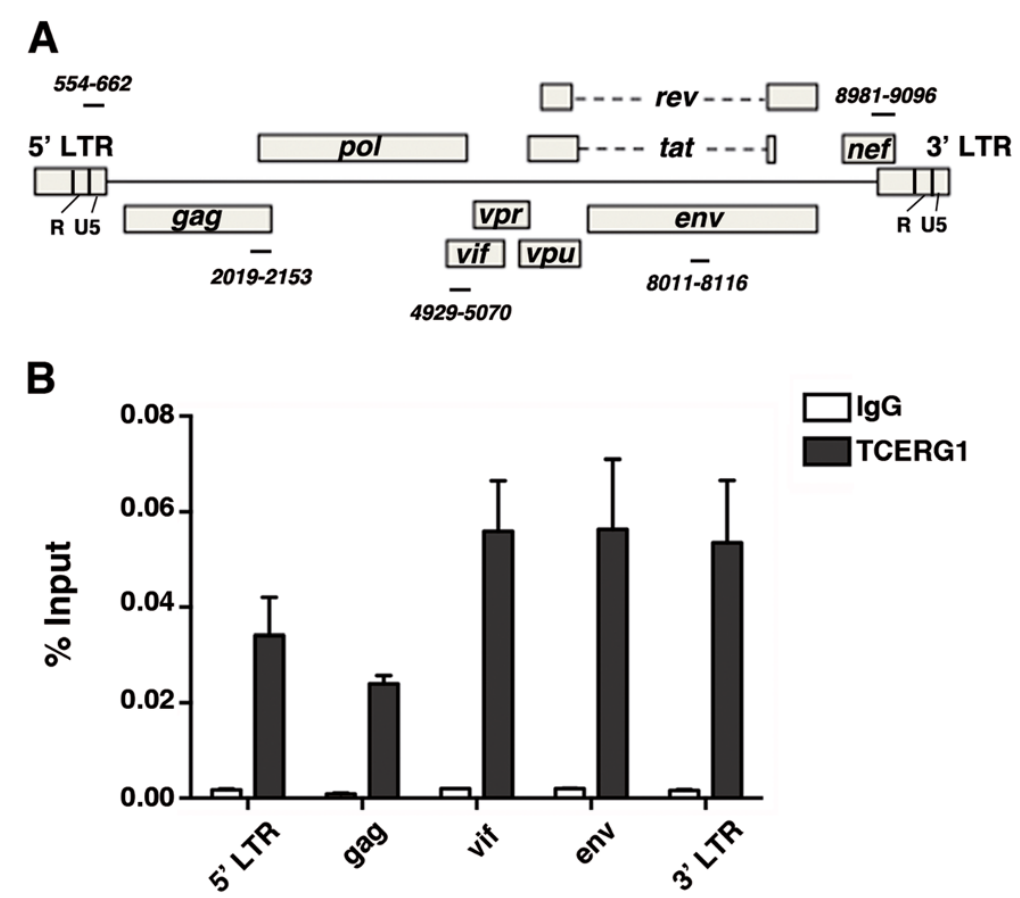

Figure 4 TCERG1 binds along the HIV-1 genome. A. Schematic representation of the HIV-1 genome. The black lines indicate the position of each pair of primers used for qChIP experiments. B. TCERG1 distribution at different positions on the HIV-1 gene detected by ChIP followed by qPCR in HEK293T cells transfected with the pNL4-3 $\triangle$ RT provirus and a TCERG1 overexpression vector. IgG was used as a control in all ChIP experiments. Data from four independent experiments are presented as percentages of the bound input.

of total RNAPII and RNAPII phosphorylated at Ser2, which was more pronounced at the distal positions of the gene (Figure 5A). No differences were observed at the promoter region upon TCERG1 knockdown. The recruitment of RNAPII phosphorylated at Ser5 also remained unaltered (Figure 5A). Conversely, TCERG1 overexpression resulted in an accumulation of total and Ser2 polymerases at the same positions (Figure 5B). These results indicate that TCERG1 is able to modify CTD phosphorylation at Ser2, which may explain how TCERG1 affects the dynamics of HIV-1 transcript elongation.

TCERG1 did not change the overall amount of Ser2 phosphorylation or total RNAPII in the cell upon TCERG1 depletion as assessed by immunoblotting analysis (Additional file 1: Figure S1A). Compensatory mechanisms to prevent loss of a protein as important as the RNAPII may occur in the stable transfectant cells. We detect a slight increase in the amount of Ser2 phosphorylation and total RNAPII upon TCERG1 overexpression (Additional file 1: Figure S1B). The latter result is in agreement with our ChIP data but caution must be taken in interpreting these results because we are measuring global Ser2 phosphorylation in the cell and our ChIP experiments of Figure 5 are restricted to the recruitment of RNAPII on the HIV-1 genome.
TCERG1 is required for HIV-1 replication

Although previous experiments have implicated TCERG1 in HIV-1 LTR regulation using transient transfection approaches, previous reports have not addressed the functional implications of these findings on viral replication in vivo. Therefore, we conducted in vivo assays to test whether TCERG1 can regulate HIV-1 replication. PBLs isolated from healthy donors or Jurkat cells were co-transfected with the HIV-1 infectious clone pNL4-3Renilla along with the pGeneClip-shTCERG1-C1 or pGeneClip-shTCERG1-3 and pGeneClip-shTCERG1-4 and/or pGeneClip-shTCERG1-1 vectors. After $18 \mathrm{~h}$, the cells were collected and lysed, and Renilla luciferase activity was measured. TCERG1 knockdown caused a more than 2-fold reduction in HIV-1 replication in PBLs and a more than 3-fold reduction in Jurkat cells (Figure 6A). The TCERG1 interference was analyzed by Western blot in both PBLs and Jurkat; $\beta$-actin was used as the loading control (Figure 6B). The co-transfection of a TCERG1 expression vector and the HIV-1 infectious clone pNL4-3-Renilla produced the opposite effect on HIV-1 replication with a more than a 2 -fold increase in the synthesis of Renilla in Jurkat cells (Figure 6C, left panel). Overexpression of TCERG1 was assessed by Western blot (Figure 6C, right panel). To lend additional support to our data, Jurkat-shTCERG1 cell lines were 
transfected with the HIV-1 infectious clone pNL4-3Renilla. HIV-1 replication was reduced in both JurkatshTCERG1-(3-4) and Jurkat-shTCERG1-(1-3-4) cell lines (Figure 6D, left panel). Finally, to corroborate the notion that the observed inhibition is a consequence of diminished viral replication, we measured the expression of the p24 viral capsid protein. Measurement of HIV-1 p24 antigen levels is a highly specific assay for monitoring HIV-1 replication and the progression of HIV-1 infection [59] and correlates with the expression of Renilla [60]. We found that expression of $\mathrm{p} 24$ was inhibited by approximately $75 \%$ in TCERG1-silenced Jurkat cells compared to control cells (Figure 6D, right panel). TCERG1 mRNA and protein interference were analyzed by RT-PCR and Western blot, respectively (Figure 6E). These data further support the results of our previous experiments indicating that TCERG1 is indeed required for HIV-1 replication in vivo. To lend additional support and specificity to our data, we tested the effect of overexpressing TCERG1 on HIV-1 replication in the Jurkat-shTCERG1 cell lines. Transient overexpression of TCERG1 in TCERG1 knockdown cells rescued HIV-1 replication (Figure 6F). Given that Tat recruits the P-TEFb complex to the RNA TAR element and activates transcription elongation through phosphorylating the RNAPII CTD, we also sought to assess the effect of CDK9 on TCERG1 depletion. To this goal, we used expression plasmids for CDK9 and a catalytically inactive CDK9 protein bearing a single amino acid change (Asp-167 to Asn) in its active domain (CDK9dn). Previous data have shown that CDK9dn is functionally able to associate in vitro and in vivo with Cyclin $\mathrm{T}$ [61]. And in Jurkat cells, overexpression of Cdk9dn specifically inhibited Tat trans-activation and HIV-1 replication [62] by preventing the endogenous
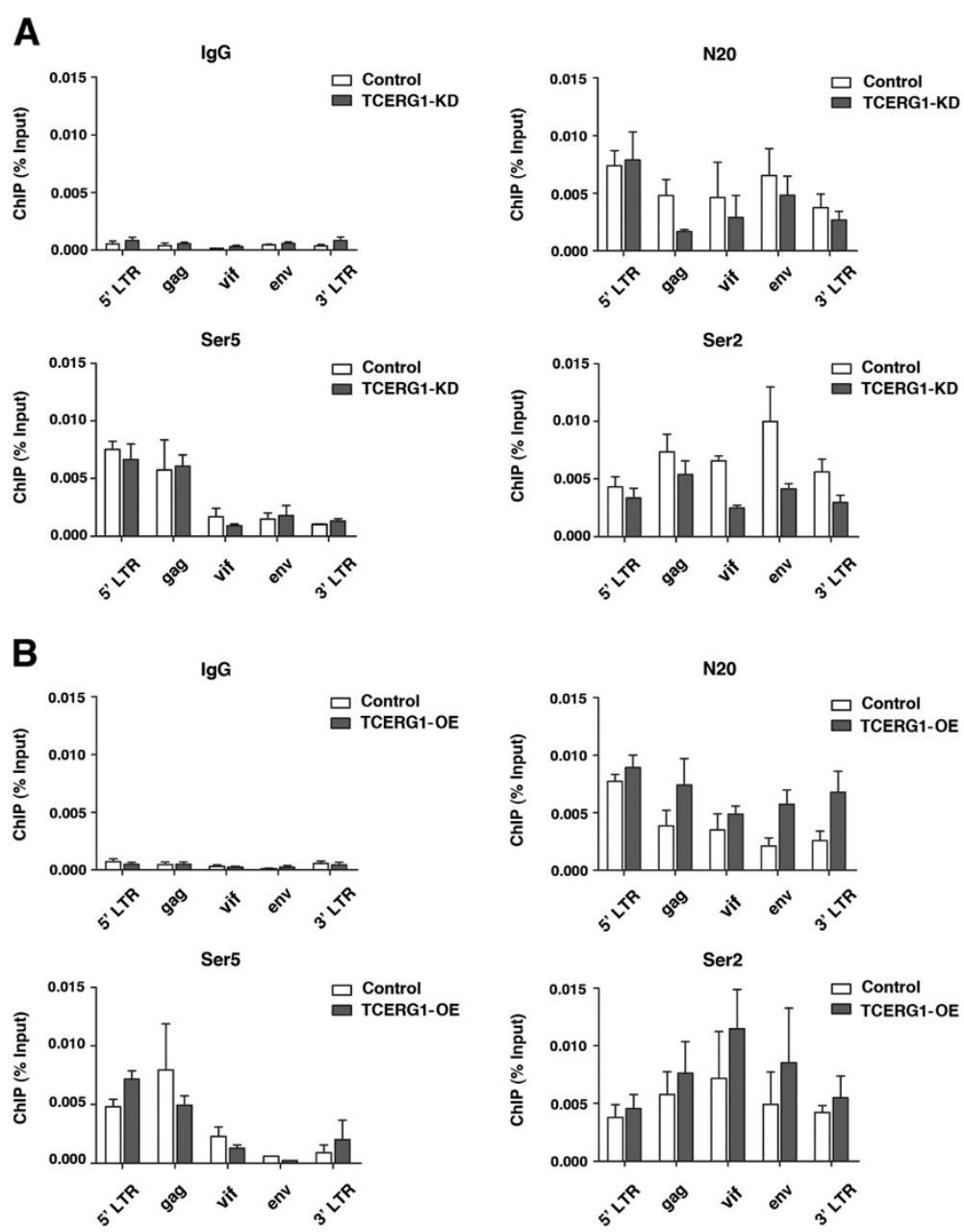

Figure 5 TCERG1 depletion decreases CTD Ser2 phosphorylation. A. Total polymerase (N20), phospho-Ser5 (Ser5) or phospho-Ser2 (Ser2) polymerase II distribution at different positions of the HIV-1 genome was detected by ChIP followed by qPCR in the control and TCERG1 knockdown (KD) T-Rex-293 cell lines transfected with the pNL4-3ART provirus. B. The same experiment described in panel $\mathbf{A}$ was performed using empty (control) and TCERG1 overexpression (OE) vectors. IgG was used as a control in all ChIP experiments. Data from four independent experiments are presented as percentages of the bound input (means \pm SEM). 


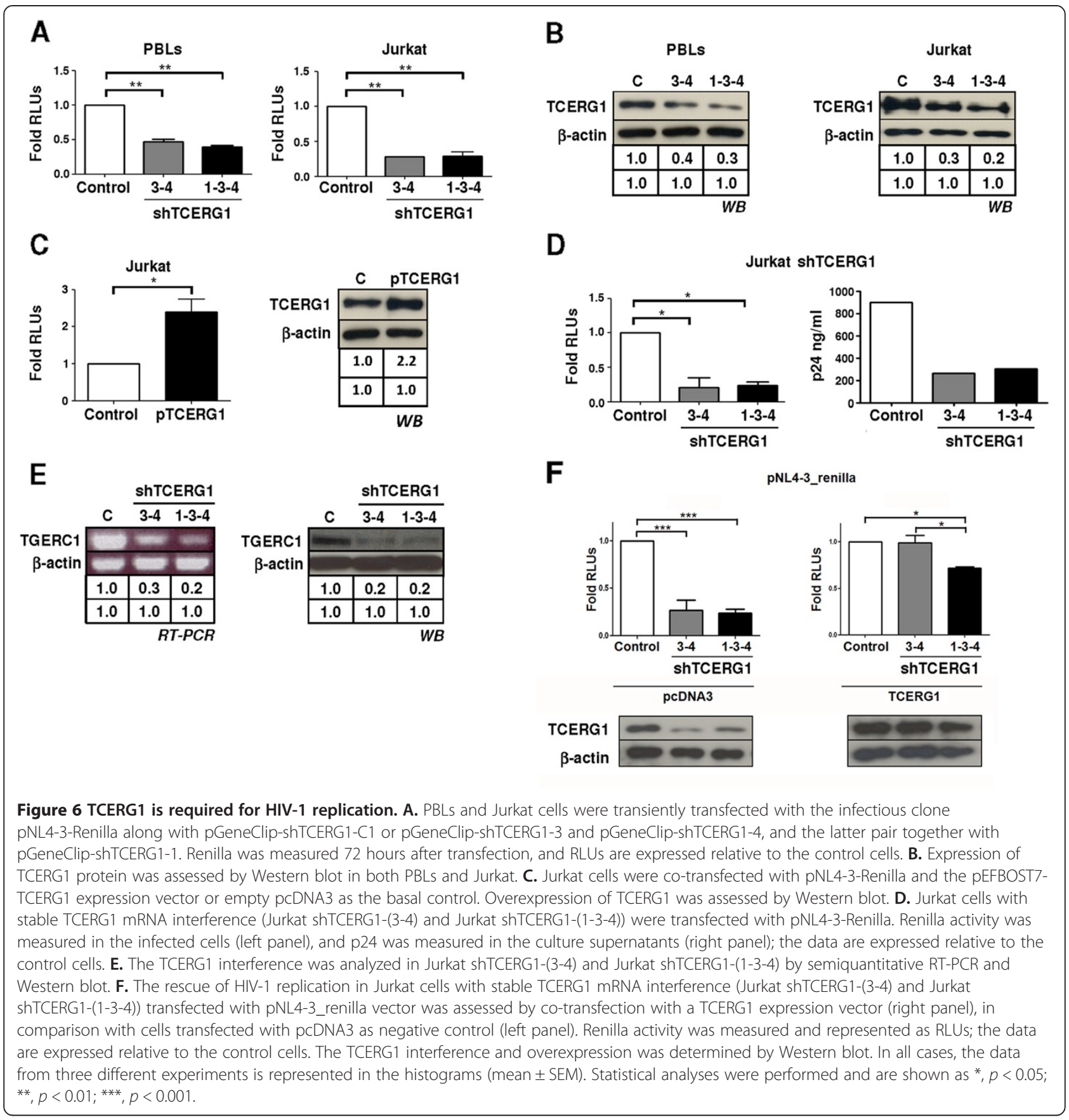

CDK9 protein from interacting with CyclinT1. Importantly, CDK9dn fails to activate viral transcription indicating that the kinase activity of CDK9 is essential for transcription activation [35,63]. Our experiments with CDK9/ CDK9dn were designed to prove that our observations were specific for HIV-1 and it could compensate (or not) for a lack of TCERG1. Therefore, the experiments were performed with a reporter LTR-HIV plasmid in order to analyze the transcriptional effects of the proteins avoiding the dominant negative effect of CDK9dn. Overexpression of CDK9 did not rescue the LTR-dependent transcription in the absence of endogenous TCERG1 (Additional file 2: Figure S2).

\section{Discussion}

Our results help to explain the mechanism by which TCERG1 functions in HIV-1 replication. We report that TCERG1 is required for both basal and Tat-dependent transcriptional activation in cells of the immune system, which are the relevant targets in HIV-1 infection. Previous 
reports have indicated that immunodepletion of TCERG1 from transcriptionally-active HeLa nuclear extracts decreases Tat activation of RNAPII elongation efficiency in vitro. However, TCERG1 does not bind directly to Tat, and the presence of TCERG1 in the Tat affinity column was later suggested to be due to the association of TCERG1 with other HIV-1 cofactors, such as P-TEFb and Tat-SF1 [51]. Based on this possibility, it was conceivable that removal of the TCERG1-associated Tat cofactors, not removal of TCERG1 per se, provoked the decrease in Tatmediated activation of transcriptional elongation. Here, we show that depletion of TCERG1 by gene silencing resulted in decreased basal and Tat-activated transcription, thus demonstrating a direct effect for TCERG1 in the regulation of HIV-1 transcription. We show previously that TCERG1 affects elongation in a promoter-specific fashion $[50,53]$ and a relatively small number of genes are affected upon TCERG1 depletion by microarray analysis [64]. Moreover, loss of tcer-1, the TCERG1 homologue in C. elegans, has little or no effect on wild-type lifespan [65]. Thus TCERG1 is not likely to be a general component of the transcriptional machinery, therefore, the most probable interpretation of our experimental data is that TCERG1 is affecting specifically to HIV-1 transcription. However, we cannot rule out the possibility of TCERG1 affecting cellular genes that are important for viral transcription.

The comparison of our results with those observed for the other HIV-1 Tat cofactor, Tat-SF1 (which was also identified by in vitro reconstituted transcription reactions with immunodepleted extracts), is interesting. In recent work, we show that depletion of Tat-SF1 by gene silencing did not affect basal or Tat-dependent transcription from an HIV-1 CAT reporter in HeLa and HEK293 cells [66]. These data contradict the results obtained by Caputi and coworkers, which have recently reported that downregulation of Tat-SF1 by siRNAs induces a decrease in transcription and Tat-mediated activation of an HIV-1 reporter minigene in HEK293 cells [67]. In this later work, the effect of reduction of TCERG1 expression was also analyzed. In contrast with our results, inhibition of TCERG1 expression partially reduced Tat-mediated trans-activation by paradoxically increasing the basal transcription of the reporter construct [67]. Our experiments with TCERG1 were predominantly performed in Jurkat and PBLs, which are often used to examine transcriptional regulation and HIV-1 infection in lymphocytes, the natural targets of HIV-1. In agreement with the results obtained in these cells, we also observed inhibition of transcription from a proviral vector upon depletion of TCERG1 in HEK293 cells (Figure 2C, Figure 2D, and other data not shown). Therefore, caution must be taken in interpreting the results obtained with Tat-SF1 and TCERG1 knockdown since different results can be obtained in different contexts. Other elements in the HIV-1 genome, the cell line used, and the chromatin environment created by the different HIV-1 plasmids could potentially contribute to the proper recruitment and functionality of these Tat cofactors.

What is the mechanism for the decrease in HIV-1 transcription upon TCERG1 depletion? Our results provide a mechanism that is based on the RNAPII elongation rate. First, we observed diminished accumulation of transcripts upon TCERG1 knockdown (Figure 2B and Figure 2C). Second, TCERG1 increased the rate of RNAPII transcription (Figure 2D). Finally, we observed that TCERG1 favors the phosphorylation of Ser2 of the RNAPII CTD (Figure 5). Based on these results, we propose that TCERG1 regulates HIV-1 transcription by increasing the phosphorylation of Ser 2 on the heptad repeats in the CTD, which facilitates the elongation of RNAPII through the DNA template during gene activation. We have taken several approaches to assess the recruitment of TCERG1 to the HIV-1 gene. Isolation of PICs assembled onto a functional HIV-1 promoter in vitro identified the presence of TCERG1 (Figure 3). ChIP analysis confirmed the presence of TCERG1 at the promoter region and distal elements (Figure 4). The recruitment of TCERG1 correlates with the modulation of Ser2 phosphorylation upon TCERG1 knockdown/ overexpression (Figure 5). These results are consistent with a role for TCERG1 in the activation of HIV-1 transcriptional elongation that leads to the release of RNAPII from promoter-proximal pausing. However we cannot exclude the possibility that TCERG1 favors RNAPII processivity by acting at other locations within the gene unit where polymerases could pause.

Very recently, a multi-component complex now called the super elongation complex (SEC) has been isolated through a sequential affinity-purification strategy to identify proteins associated with both Tat and P-TEFb [68]. In addition to Tat and P-TEFb, the SEC contains the transcription factors ELL2, AFF4, ENL, and AF9. The combined action of Tat, $\mathrm{P}-\mathrm{TEFb}$ and the SEC results in the synergistic activation of HIV-1 elongation by helping to release RNAPII from promoter-proximal pausing and promote the production of full-length viral transcripts $[68,69]$. ELL2 is a well-characterized transcription elongation factor and member of the ELL family of transcription factors that stimulate elongation by increasing the catalytic rate and suppressing transient pausing of RNAPII [70,71]. Strikingly, we have identified ELL as a TCERG1interacting protein using a tandem affinity purification (TAP) strategy (data not shown). Our data show that overexpression of CDK9 did not rescue the HIV-1 LTRdependent transcription in the absence of endogenous TCERG1 (Additional file 2: Figure S2). Based on these observations, it is an exciting possibility that TCERG1 affects 
RNAPII processivity via a mechanism that involves the recruitment and/or assembly of functional transcription complexes.

One novel finding presented here is that TCERG1 is required for HIV-1 replication (Figure 6). This requirement is likely due to the effect of TCERG1 on viral transcription, although the precise mechanism by which this factor functions remains unknown. Early data that suggested a role for TCERG1 in HIV-1 replication found that the levels of TCERG1 increased in Jurkat T cells cultured with CXCL12 or the HIV-1 glycoprotein gp120 IIIB [72]. This expression was found to be dependent on the presence of the HIV-1 co-receptor CXCR4 [72]. Recently, several studies using genome-wide RNAi experiments have identified the so-called HIV dependency factors (HDFs) [73-75]. TCERG1 was not identified in any of these studies. However, the three siRNA screens showed little overlap at the level of individual genes, perhaps due to differences in the cell lines and HIV strains used, the assay time post-infection or the procedures used to measure infection. A recent work exploiting the interactions identified in these RNAi screens in the context of a human protein-protein interaction network has predicted undiscovered HDFs that may play a role in HIV-related disease progression in lymph nodes. Importantly, TCERG1 was one of the newly predicted HDFs in this study [76].

\section{Conclusions}

Although we have gained a considerable knowledge regarding the early events that occur at the promoter region of genes to trigger the release of RNAPII for elongation, several recent discoveries have suggested that events subsequent to transcript initiation may also be necessary for the production of full-length transcripts. Specifically, the rate of elongation and pausing of RNAPII are generating considerable interest as critical regulators to ensure precise and reliable control of gene expression. In this scenario, factors that affect these processes could act as checkpoint regulators to guarantee optimal RNAPII activity. In this work, we describe the nuclear factor TCERG1 as positive regulator of HIV-1 transcriptional elongation by increasing the elongation rate of RNAPII and phosphorylation of Ser 2 within the CTD. TCERG1 is therefore a candidate checkpoint factor that could mediate functional links between elongation rates and pausing.

The experiments reported here demonstrate an important role for TCERG1 in controlling HIV-1 transcriptional elongation and replication. These studies suggest that TCERG1 might be a plausible new target for antiretroviral therapy, thus opening interesting new avenues for future investigation.

\section{Methods}

\section{Plasmids}

The pNL4-3 wild-type (wt) plasmid, which contains the complete HIV-1 genome and induces infectious progeny after transfection, was kindly provided by Dr M.A. Martin [77]. The pNL4-3- $\Delta \mathrm{RT}$ plasmid was created by deleting the reverse transcriptase gene of the pNL4-3 wt plasmid and was kindly provided by Dr SánchezPalomino (Hospital Clínic, Barcelona) [78]. The pNL43-Renilla plasmid was obtained by replacing the nef gene in the HIV-1 proviral clone pNL4-3 with the Renilla luciferase gene, as previously described [60]. The LTR-LUC plasmid containing the luciferase (LUC) reporter gene under the control of the HIV-1 LTR $\mathrm{U} 3+\mathrm{R}$ region (LAI strain) has previously been described [79]. The pCMVTat101 plasmid has previously been described [80]. The eukaryotic expression pEFBOST7-TCERG1 plasmid has been previously described [50]. CDK9 and CDK9dn expression plasmids were described previously [35]. The GeneClip U1 Hairpin Cloning System kit (Promega Biotech Iberica, Madrid, Spain), which contains the pGeneClip vector, was used to generate the following small hairpin RNA (shRNA) plasmids: pGeneClip-shTCERG1-1, pGeneClip-shTCERG1-3, and pGeneClip-shTCERG1-4) containing three different small interference RNA (siRNA) sequences directed against the mRNA encoding for TCERG1. The sequences used to generate the pGeneClipshTCERG1-1 plasmid were shTCERG1-1 s (5' ${ }^{\prime}$-TCTCGA AGGAGTTGCACAAGATAAAGTTCTCTTATCTTGTG CAACTCCTTCCT-3') and shTCERG1-1 as (5' -CTGCAG GAAGGAGTTGCACAAGATAAGAGAACTTTATCTTG TGCAACTCCTTC-3'). The sequences used to generate the pGeneClip-shTCERG1-3 plasmid were shTCERG1-3 s (5' TCTCGATCCTCGATGTATTAAGTAAGTTCTCTACTT AATACATCGAGGATCCT-3') and shTCERG1-3as (5' -CT GCAGGATCCTCGATGTATTAAGTAGAGAACTTACT TAATACATCGAGGATC-3'). The sequences used to generate the pGeneClip- shTCERG1-4 plasmid were shT CERG1-4 s (5' - TCTCGGCATGACTGACATACATAAA GTTCTCTTATGTATGTCAGTCATGCCCT-3') and shT CERG1-4as (5' -CTGCAGGGCATGACTGACATACATA AGAGAACTTTATGTATGTCAGTCATGCC-3'). The scrambled sequences used to generate the pGeneClip-shT CERG1-C1 plasmid were shTCERG1-C1s (3'-TCTCGG ACTGGAAGTTCAAAGAAAAGTTCTCTTTCTTTGAA CTTCCAGTCCCT-5') and shTCERG1-C1as (3'-CTGC AGGAGAGGCGTTTTAGAATATAGAGAACTTATATT CTAAAACGCCTCTC-5'). Each pair of primers were annealed and cloned in the linearized pGeneClip vector according to the manufacturers' instructions. The pEGFP-C1 vector, which was used as control for monitoring transfection efficiency, was purchased from Clontech (Mountain View, CA). Plasmids were purified using the QIAGEN Plasmid Maxi Kit (Qiagen Iberia, 
Madrid, Spain) following the manufacturer's instructions. The human growth hormone-expressing plasmid pXGH5 was kindly provided by Dr Heiner Schaal (University of Düsseldorf, Germany). The pEYFP-C1 vector (BD Biosciences, Clontech) was co-transfected as a control for monitoring transfection efficiency and was measured using a FACSCalibur flow cytometer (BD Biosciences, Clontech).

\section{Cells}

The Jurkat cell line was cultured in RPMI 1640 medium (BioWhittaker, Walkersville, MD) supplemented with 10\% fetal calf serum (PAN Biotech $\mathrm{GmbH}$, Aidenbach, Germany), $2 \mathrm{mM}$ L-glutamine, $100 \mu \mathrm{g} / \mathrm{ml}$ streptomycin and $100 \mathrm{U} / \mathrm{ml}$ penicillin (Lonza, Basel, Switzerland) at $37^{\circ} \mathrm{C}$. PBMCs were isolated from the blood of at least three different healthy donors by centrifugation through a Ficoll-Hypaque gradient (Lymphocyte Separation Medium, Lonza). The adherent monocytes were eliminated by keeping the culture flask horizontal for 1 hour and then separating the non-adherent lymphocytes. These peripheral blood lymphocytes (PBLs) were collected in supplemented RPMI 1640 medium and maintained at $2 \times 10^{6}$ cells $/ \mathrm{ml}$ at $37^{\circ} \mathrm{C}$.

Two Jurkat cell lines with stably interference for TCERG1 were produced: Jurkat shTCERG1-(3-4), which was co-transfected with both plasmids pGeneClip-shT CERG1-3 and pGeneClip-shTCERG1-4 (1:1); and Jurkat shTCERG1-(1-3-4), which was co-transfected with plasmids pGeneClip-shTCERG1-1, pGeneClip-shTCERG1-3, and pGeneClip-shTCERG1-4 (1:1:1). The control cells Jurkat shTCERG1-C1 were Jurkat stably transfected with the control vector pGeneClip-shTCERG1-C1. All three cell lines were grown in supplemented RPMI 1640 medium with $5 \mu \mathrm{g} / \mathrm{ml}$ puromycin (Invitrogen, Barcelona, Spain) at $37^{\circ} \mathrm{C}$.

HEK293T cells were grown in DMEM (Dulbecco's modified Eagle Medium, Gibco) with 10\% fetal bovine serum (Gibco) supplemented with penicillin/streptomycin (100U and $0.1 \mathrm{mg} / \mathrm{ml}$, respectively) and $4 \mathrm{mM} \mathrm{L-}$ glutamine (Gibco).

The Flp-In T-Rex-293 cell line is a tetracycline-inducible mammalian expression system (Invitrogen). Cells were maintained in high glucose DMEM with $10 \%$ fetal bovine serum supplemented with penicillin/streptomycin, $4 \mathrm{mM}$ L-glutamine, and $15 \mu \mathrm{g} / \mathrm{ml}$ blasticidin (Invitrogen) to select for cells expressing the Tet repressor plasmid. To create inducible shRNA expressing cell lines, hairpin sequences targeting either GFP (control) or TCERG1 were cloned into pcDNA5/FRT/TO plasmid (Invitrogen) which contains a CMV promoter with two tandem repeats of the Tet operator. These constructs were transfected in the Flp-In T-Rex-293cells along with pOG44 which expresses the Flp recombinase. Stably transfected cells were selected with $200 \mu \mathrm{g} / \mathrm{mL}$ hygromycin and $15 \mu \mathrm{g} / \mathrm{mL}$ blasticidin. $5 \mu \mathrm{g} / \mathrm{mL}$ tetracycline (Sigma) was used for the induction of shRNA expression.

\section{Reagents and antibodies}

IL-2 was used at $300 \mathrm{U} / \mathrm{ml}$ (Chiron, Emeryville, CA). Propidium Iiodide and 4',6-diamidino-2-phenylindole (Dapi) were obtained from Sigma-Aldrich. Specific antibodies against TCERG1 have previously been described [51]. Monoclonal antibody against HIV-1 Tat (aa 2-9) was obtained from Advanced Biotechnologies Inc. (Columbia, $\mathrm{MD})$. Specific antibodies against the $\beta$-isoform of actin were obtained from Sigma-Aldrich. Antibodies against CDK9 (C-20, sc-484), and RNAPII (N20, sc-899), TBP (N12, sc-204), CDK2 (M2, sc-163), and nucleolin (C23, sc-13057) were obtained from Santa Cruz Biotechnology. Anti-S5 and anti-S2 antibodies were obtained from Abcam. Secondary antibodies conjugated to horseradish peroxidase (HRP) were purchased from GE Healthcare Spain (Madrid, Spain). Secondary antibodies conjugated to Alexa-488 and Alexa-546 were purchased from Molecular Probes (Eugene, OR).

\section{Transfection assays}

Stable transfection of Jurkat cells with pGeneClip-shT CERG1-C1 or pGeneClip-shTCERG1-3 and pGeneClipshTCERG1-4 and/or pGeneClip-shTCERG1-1 vectors was performed by electroporation with an Easyjet Plus Electroporator (Equibio, Middlesex, UK). In brief, $5 \times 10^{6}$ cells were collected in $250 \mu \mathrm{l}$ of RPMI without supplements and mixed with $5 \mu \mathrm{g}$ of DNA in an electroporation cuvette with a $2 \mathrm{~mm}$ electrode gap (Equibio). The cells were transfected by two pulses at $280 \mathrm{~V}, 150 \mu \mathrm{F}$, and $330 \Omega$. After transfection, the cells were incubated in supplemented RPMI for $18 \mathrm{~h}$ at $37^{\circ} \mathrm{C}$. Puromycin was then added to the culture medium at $0.5 \mu \mathrm{g} / \mathrm{ml}$ to select stable shRNA expressing cells. For transient transfections, approximately $5-10 \times 10^{6}$ cells were resuspended in $350 \mu \mathrm{l}$ of RPMI without supplements and mixed with 5-10 $\mu \mathrm{g}$ of DNA in an electroporation cuvette with a $4 \mathrm{~mm}$ electrode gap (Equibio). Jurkat cells were transfected by one pulse at $280 \mathrm{~V}$ and $1500 \mu \mathrm{F}$ and resting PBLs were transfected by one pulse at $320 \mathrm{~V}$ and $1500 \mu \mathrm{F}$. After transfection, the cells were incubated in supplemented RPMI for $18 \mathrm{~h}$ at $37^{\circ} \mathrm{C}$. The HIV-1 p24 antigen was measured in cell culture supernatants by an enzyme-like immunoassay (InnotestTM HIV Ag mAb; Innogenetics, Barcelona, Spain). Luciferase and Renilla activities were assayed using the Luciferase Assay System (Promega), and transfection efficiency was monitored by flow cytometry to measure the quantity of cells expressing EYFP. Relative luciferase and Renilla units (RLUs) were measured in supernatants with a Sirius luminometer (Berthold Detection Systems, Oak Ridge, TN) after addition of the appropriate 
substrate. RLUs were normalized by measuring both $\beta$ Galactosidase activity and total protein concentration using the Bradford method [81].

For the RNA interference (RNAi) knockdown experiments, HEK293T cells were grown in $60-\mathrm{mm}$ plates (Falcon) to $70-80 \%$ confluence and transfected using Lipofectamine 2000 reagent (Invitrogen) according to the manufacturers' protocol with $60 \mathrm{nM}$ of either one of the following small interfering RNA (siRNA) duplexes: siEGFP, 5'-CUACAACAGCCACAACGE-3', or siTCERG1, 5' -GGAGUUGCACAAGAUAGUU-3' [53]. Cells that did not receive siRNA were also used as a control. After $48 \mathrm{~h}$, the plasmids pNL4-3- $\Delta \mathrm{RT}$ and pXGH5 were co-transfected using Lipofectamine 2000. Cells were harvested $24 \mathrm{~h}$ later. Under these conditions, we consistently achieved at least $\sim 80 \%$ knockdown of TCERG1.

\section{RNA extraction and RT-PCR assays}

Total RNA was isolated using the RNeasy Mini kit (Qiagen) following manufacturer's instructions. Specific primers for amplifying a $431 \mathrm{bp}$ fragment of the 3 '-end of the tcerg1 gene were as follows: TCERG1-s, 5'-AAGGCC CGTTCAGAACAAACA-3'; and TCERG1-as, 5'-CGTCC TGAAGTCAGCTTTGGCT-3'. Primers for amplifying the human $\beta$-actin gene were as follows: $\beta$-actin-1 s, 5'-TCACCCACACTGTGCCCATCTA-3', and $\beta$-actin1as, 5 '-AGTTGAAGGTAGTTTCGTGGAT-3', yielding a fragment of $360 \mathrm{bp}$. The RT-PCR assay was performed in a C1000 Thermal Cycler (Bio-Rad Laboratories, Madrid, Spain) using the Access RT-PCR System (Promega). Briefly, the reaction mix contained $5 \mu \mathrm{l}$ of $5 \mathrm{x}$ buffer, $2 \mathrm{mM} \mathrm{MgCl} 2,0.1 \mathrm{mM}$ dNTPs, $0.4 \mu \mathrm{M}$ of each primer pair, and $0.5 \mu \mathrm{l}$ of each AMV Reverse Transcriptase and Tfl DNA Polymerase in a final volume of $25 \mu \mathrm{l}$. A total of $3 \mu \mathrm{g}$ of RNA was added, and the thermal cycling conditions were as follows: an initial cycle of $48^{\circ} \mathrm{C}$ for $45 \mathrm{~min}$ and $95^{\circ} \mathrm{C}$ for $10 \mathrm{~min} ; 29$ cycles of $95^{\circ} \mathrm{C}$ for $30 \mathrm{sec}, 51^{\circ} \mathrm{C}$ for $1 \mathrm{~min}, 68^{\circ} \mathrm{C}$ for $45 \mathrm{sec}$; and a final extension cycle of $68^{\circ} \mathrm{C}$ for $10 \mathrm{~min}$. Amplified products were analyzed by electrophoresis on a $3 \%$ SeaKem agarose gel containing $5 \mu \mathrm{g} / \mathrm{ml}$ of ethidium bromide in $0.5 \mathrm{x}$ Tris-acetate buffer. The specificity of the amplified products was assessed by sequencing using an $\mathrm{ABI}$ Prism 3700 DNA Analyzer (Applied Biosystems, Foster City, CA).

For the analysis of the HIV genome elongation products (Figure 2B), the Jurkat-shTGERC1-(3-4), Jurkat-shTGE RC1-(1-3-4) and Jurkat-shTGERC1-C1 cells were transfected with an infectious HIV-1 pNL4-3-wt clone and cultured for $48 \mathrm{~h}$. Total RNA was isolated using the RNeasy Mini kit (Qiagen) following the manufacturer's instructions, and $5 \mu \mathrm{g}$ of RNA was used to synthesize
cDNA with the GoScript Reverse Transcription System (Promega). Transcript elongation products were analyzed using SYBR Green PCR Master Mix in an ABI-Applied Biosystems 7500 Real Time Thermal Cycler (Applied Biosystems). The reaction mixture contained $10 \mu \mathrm{l}$ of the PCR Master Mix and 0.4 $\mu \mathrm{M}$ of each primer pair in a final volume of $20 \mu \mathrm{l}$. One microliter of cDNA was added and amplified using the following conditions: an initial 10 min denaturation step at $95^{\circ} \mathrm{C}$ and 36 cycles consisting of $95^{\circ} \mathrm{C}$ for $15 \mathrm{sec}, 60^{\circ} \mathrm{C}$ for $1 \mathrm{~min}$, followed by a continuous melting curve stage. The following primers were used to amplify an early elongation product, yielding a fragment of $303 \mathrm{bp}: \mathrm{R} / \mathrm{U} 5$ (sense), 5' GGCTAACTAGGGAACCCACTGCTT-3' (496-519) and gag (antisense), 5'-CTCGCACCCATCTCTCTCCTTCT A-3' (777-799). To amplify a late elongation product, at the beginning of the nef gene, primers $\mathrm{P} 3$ (sense), 5'TTGCTCAATGCCACAGCCAT-3' (8654-8673) and P4 (antisense), 5' -TTTGACCACTTGCCACCCAT-3' (87878806) [82] were used and a fragment of 152 bp was produced. Amplification of the gene encoding $\beta$-actin was used as an internal control for data normalization using the following primers: $\beta$-actin-2 s, 5'-AGGCC CAGAGCAAGAGAGGCA-3'; $\beta$-actin-2as, $5^{\prime}$-CGCAG CTCATTGTAGAAGGTGTGGT-3', yielding a fragment of $114 \mathrm{bp}$. All amplified products were analyzed by electrophoresis on agarose gels and the specificity of amplified products was assessed by sequencing. Densitometry analysis was performed using Quantity One software (Bio-Rad). Gel bands were quantified and background noise was subtracted from the images. The relative ratio of optical density units was calculated relative to the gel band corresponding to the internal control for each lane and each type of RNA sample.

For the experiment shown in Figure 2C, total RNA was extracted from cells grown in 60-mm plates (Falcon) as previously described [53]. Approximately $3 \mu \mathrm{g}$ of digested RNA was reverse transcribed using random hexamers. The quantification of the transcripts derived from the pNL4-3- $\Delta$ RT plasmid was performed by realtime PCR using the PerfeCTa SYBR Green SuperMix for iQ (Quanta Biosciences) and the iCycler thermal cycler station (Bio-Rad) with the following primers: ENV-F, 5' TGGAAAACTCATTTGCACCA-3' (8011-8027) and EN V-R, 5'-TTCTCTGTCCCACTCCATCC-3' (8097-8116). Glyceraldehyde-3-phosphate dehydrogenase (GAPDH) was used as an internal control and was amplified using the following primers: GAPDHfwd, 5'-ATGGGGGAA GGTGAAGGTCG-3' and GAPDHrev, 5'-GGGTCATT GATGGCAACAATATC-3'. pXGH5 was used as a transfection control and its products were amplified with the primers hGH-fwd, 5'-CAACAGAAATCCAACC TAGAGCTGCT-3' and hGH-rev, 5' -TCTTCCAGCCT CCCATCAGCGTTTGG-3' . 


\section{Purification of PICs and in vitro transcription/elongation assay}

The purification of PICs and the in vitro transcription/ elongation assay were performed as previously described [35].

\section{Immunoblotting assays}

Protein extracts were obtained as previously described [83]. Protein concentration was determined by the Bradford method. Forty micrograms of protein extracts were fractionated by sodium dodecyl sulfate-polyacrylamide gel electrophoresis (SDS-PAGE) and transferred onto Hybond-ECL nitrocellulose paper (GE Healthcare). After blocking and incubation with primary and secondary antibodies, proteins were detected with SuperSignal West Pico/Femto Chemiluminescent Substrate (Pierce, Rockford, IL). Images were acquired using a BioRad Gel Doc 2000 (Bio-Rad). Densitometry was performed as described above. For the experiment shown in Figure 4A, we used protocols that have previously been described [53].

\section{Immunofluorescence assays}

For immunofluorescence assays, cells were immobilized in PolyPrep slides (Sigma-Aldrich) for 15 minutes and then fixed with $2 \%$ paraformaldehyde (PFA)-0.025\% glutaraldehyde in PBS for 10 minutes at room temperature. After washing twice with $0.1 \%$ glycine/PBS, cells were permeabilized with $0.1 \%$ Triton $\mathrm{X}-100 / \mathrm{PBS}$ for 10 minutes. Cells were then treated with $1 \mathrm{mg} / \mathrm{ml} \mathrm{NaBH} 4$ for $10 \mathrm{mi}$ nutes. Incubation for 1 hour at room temperature with each primary and secondary antibodies and subsequent washes were performed with PBS1x-2\% BSA-0.05\% saponine buffer. Coverslips were immobilized with $70 \%$ glycerol/PBS. Images were obtained with a Radiance 2100 confocal microscope (BioRad, Hercules, CA), Leica TCS-SP confocal microscope (Leica Microsystems, Wetzlar, Germany) or a Leica DMI 4000B Inverted Microscope (Leica Microsystems).

\section{Chromatin immunoprecipitation assay}

Control and TCERG1 knockdown T-Rex-HEK293 cells were seeded in 100-mm diameter plates at 70-80\% confluence and transfected with $6 \mu \mathrm{g}$ of pNL4-3 $\Delta \mathrm{RT}$ plasmid. For overexpression experiments, T-Rex-HEK293 cells were additionally transfected with the pEFBOST7 empty vector or pEFBOST7-TCERG1 expression vector using the calcium phosphate precipitation method. After $48 \mathrm{~h}$, the cells were fixed with $1 \%$ formaldehyde and incubated at room temperature for $10 \mathrm{~min}$. The cross-link was arrested by adding glycine $(0.125 \mathrm{M})$ for an additional $5 \mathrm{~min}$ at room temperature. Subsequently, the cells were pelleted, washed 3 times with phosphatebuffered saline (PBS), and lysed in SDS lysis buffer (1\% SDS, $10 \mathrm{mM}$ EDTA, $50 \mathrm{mM}$ Tris- $\mathrm{HCl}$ [pH 8.1], protease inhibitor mixture [Complete; Roche], and $1 \mathrm{mM}$ phenylmethylsulfonyl fluoride [PMSF]) for $10 \mathrm{~min}$ on ice. The lysates were sonicated 8 times for $15 \mathrm{~s}$ on ice and centrifuged at maximum speed. The sheared chromatin was diluted by the addition of 10 volumes of ChIP buffer (0.01\% SDS, 1.1\% Triton X-100, 1.2 mM EDTA, $16.7 \mathrm{mM}$ Tris- $\mathrm{HCl}$ [pH 8.1], $167 \mathrm{mM} \mathrm{NaCl}$, protease inhibitor mixture, and $1 \mathrm{mM}$ PMSF) and pre-cleared with a salmon sperm DNA/protein A-agarose fast-flow slurry (Millipore) for $2 \mathrm{~h}$. The beads were removed by centrifugation. $5 \%$ of the pre-cleared chromatin was removed to serve as the pre-immunoprecipitation (pre-IP; input) control, and the remaining pre-cleared chromatin was incubated overnight with $5 \mu \mathrm{g}$ of the specific antibodies or nonspecific rabbit IgG. The chromatin-antibody complexes were collected by incubation with salmon sperm DNA/protein-A agarose (50\% slurry) and centrifugation. Beads were washed in low or high salt conditions using buffer $(0.1 \%$ SDS, $1 \%$ Triton $\mathrm{X}-100,2 \mathrm{mM}$ EDTA, $20 \mathrm{mM}$ Tris- $\mathrm{HCl}$ [pH 8.1], and $150 \mathrm{mM} \mathrm{NaCl}$ ) containing $20 \mathrm{mM}$ and $500 \mathrm{mM} \mathrm{NaCl}$, respectively. The beads were then washed once with $\mathrm{LiCl}$ buffer $(0.25 \mathrm{M} \mathrm{LiCl}, 1 \% \mathrm{NP}-40,1 \%$ Na-deoxycholate, $1 \mathrm{mM}$ EDTA, and $10 \mathrm{mM}$ Tris- $\mathrm{HCl}$ [pH 8.0]) followed by two washes with Tris-EDTA buffer. The antibody-chromatin complexes were eluted from the beads by incubation with elution buffer $(0.1 \% \mathrm{SDS}, 0.1 \mathrm{M} \mathrm{NaHCO}$ ). A final concentration of $0.2 \mathrm{M} \mathrm{NaCl}$ was added to eluates, and the samples were incubated at $65^{\circ} \mathrm{C}$ for 4-6 h. The samples were treated with RNase $A$ and proteinase $K$, and the DNA was purified using phenol-chloroform extraction. The pre-IP input sample was purified in a similar manner. The DNA obtained was amplified by quantitative PCR (qPCR) using PerfeCTa SYBR Green SuperMix for iQ (Quanta Biosciences). The following primers were used: 5'-TAGTGTGTGCCCGTCTGTTG-3 (554-573) and 5'CGCTTTCAAGTCCCTGTTCG-3' (643-662) for the 5' LTR; 5' -AAAGGGCTGTTGGAAATGTG-3' (2019-2038) and 5'-GGCTCTGGTCTGCTCTGAAG-3' (2131-2153) for the $g a g$ gene; 5 '-GTTTGGAAAGGACCAGCAAA-3' (4929-4948) and 5'-CACAATCATCACCTGCCATC-3' (5051-5070) for the vif gene; 5' -TGGAAAACTCATTTG CACCA-3' (8011-8027) and 5'-TTCTCTGTCCCACTC CATCC-3' (8097-8116) for the env gene; 5'-GGTGGGT TTTCCAGTCACAC-3' (8981-9000) and 5'-GGGAGT GAATTAGCCCTTCC-3' (9077-9096) for the 3' LTR. Dilutions of the input were used to normalize the obtained values. The results represent the average of four independent experiments.

\section{RNAPII processivity assay}

The rates of RNAPII transcription were measured as previously described [53,54] with minor modifications. Briefly, control and TCERG1 knockdown T-Rex-HEK293 cells were grown to approximately 70 to $80 \%$ confluence 
and transfected with $1 \mu \mathrm{g}$ of pNL4-3- $\Delta \mathrm{RT}$ and pXGH5 plasmids using Lipofectamine 2000 (Invitrogen) according to the manufacturers' protocol. The next day, the cells were treated with $100 \mu \mathrm{M}$ 5,6-Dichlorobenzimidazole $1-\beta-D$-ribofuranoside (DRB) (Sigma) for $3 \mathrm{~h}$. The cells were washed with PBS to remove the DRB and incubated in fresh medium for various periods of time. Total RNA was then isolated using TRIzol reagent. The cDNA was amplified by qPCR using the PerfeCTa SYBR Green SuperMix for iQ (Quanta Biosciences) with primers ENV-F and ENV-R. GAPDH was used as a reference gene control and amplified with GAPDHfwd and GAPDHrev. Amplification of pXGH5 with the primers hGH-fwd and hGH-rev was used as transfection control. The $C T$ was calculated as follows: $(E$ pNL4-3- $\Delta \mathrm{RT})$ $\triangle C T \mathrm{pNL} 4-3-\triangle \mathrm{RT} /(E \mathrm{GAPDH}) \Delta C T$ GAPDH , where $E$ is the PCR efficiency and $\Delta C_{T}=C_{T}$ for the control $-C_{T}$ for DRB-treated cultures.

\section{Statistical analysis}

Statistical analysis was performed using Graph Pad Prism 5.0 (Graph Pad Software Inc., San Diego, CA) with oneway analysis of variance (ANOVA) followed by Bonferroni post-test analysis to describe the significant differences between groups. $p$-values $(p)<0.05$ were considered statistically significant in all comparisons and are represented as *, ** or *** for $p<0.05, p<0.01$ or $p<0.001$, respectively.

\section{Additional files}

Additional file 1: Figure S1. Effect of silencing and overexpression of TCERG1 on the expression and phosphorylation of RNAPII. Expression of RNAPII and its phosphorylation on ser2 was analyzed by Western blot in Jurkat cells with stable TCERG1 mRNA interference (Jurkat shTCERG1-(3-4) and Jurkat shTCERG1-(1-3-4)), in comparison with control cells Jurkat shTCERG1-C1 (A) and in Jurkat cells with transient overexpression of TCERG1, in comparison with cells transfected with an empty pCDNA3 vector (B). $\beta$-actin was used as loading control.

Additional file 2: Figure S2. Overexpression of CDK9 did not rescue the LTR-dependent transcription in the absence of TCERG1. Jurkat cells with stable TCERG1 mRNA interference (Jurkat shTCERG1-(1-3-4)) were cotransfected with LTR-LUC and a CDK9 expression vector (wild-type (wt) or mutant (dn)) (right panel), in comparison with control cells Jurkat shTCERG1-C1 (left panel). Luciferase activity was measured and represented as RLUs; the data are expressed relative to the cells transfected with pCDNA3. The CDK9 overexpression was determined by Western blot. In all cases, the data from three different experiments is represented in the histograms (mean \pm SD). Statistical analyses were performed and are shown as $*, p<0.05 ; * *, p<0.01 ; * *, p<0.001$.

\section{Abbreviations}

TCERG1: Transcription elongation regulator factor 1; RNAPII: RNA polymerase II; CTD: Carboxyl-terminal domain; LTR: Long terminal repeat; Ser: Serine; PICs: Pre-initiation complexes.

\section{Competing interests}

The authors declare that they have no competing interests.

\section{Authors' contributions}

MC, CHM, JA, and CS conceived the study. MC, MM, IM, MRLH, and EM performed the experiments. CLS and MAGB generated the Flp-In T-Rex-293 cell lines used in the analyses. All authors contribute in writing the paper. All authors have read and approved the submission of the manuscript.

\section{Acknowledgments}

This work was supported by grants from the Spanish Ministry of Science and Innovation (BFU2011-24577), the Foundation for Research and Prevention of AIDS in Spain (FIPSE-36768/08), and the Andalusian Government (Excellence Project CVI-4626/2009) to C.S.; by the Spanish Ministry of Science and Innovation (BFU2009-08796), and the Andalusian Government (Excellence Project CTS-6587) to C.H.M; and by FIPSE (360924/10), the Spanish Ministry of Economy and Competitiveness (SAF2010-18388; FIS PI0120506), the Spanish Ministry of Health (EC11-285, -278), Instituto de Salud Carlos III, AIDS Network ISCIII-RETIC (RD12/0017/0015), and the Health Programme 2009 on Combined Highly Active Anti-Retroviral Microbicides (CHAARM) to M.C. and J.A. Support from the European Region Development Fund, ERDF (FEDER) is also acknowledged. M.M. was supported by a fellowship from the Spanish Ministry of Education (FPU program). M.R.L.H was supported by a fellowship from the European Union (CHAARM). C.L.S. was funded by a fellowship from the Foundation for Medical Research (F.R.M., France) and by funds from NIH RO1 GM071037 (USA) to M.A.G-B.

We are grateful to members of the laboratories for their helpful suggestions, critical discussions, and comments. We also appreciate the secretarial assistance of Mrs Olga Palao. We thank Centro de Transfusiones from Autonomous Community of Madrid, Spain, for providing the buffy coats.

\section{Author details}

'AIDS Immunopathology Unit, Centro Nacional de Microbiología, Instituto de Salud Carlos III, Majadahonda, Madrid, Spain. ${ }^{2}$ Department of Molecular Biology, Instituto de Parasitología y Biomedicina "López Neyra" (IPBLN-CSIC), Armilla, Granada 18016, Spain. ${ }^{3}$ Department of Molecular Genetics and Microbiology, and Center for RNA Biology, Duke University Medical Center, 213 Research Drive, Durham, NC 27710, USA. ${ }^{4}$ Department of Cell Biology and Immunology, Instituto de Parasitología y Biomedicina "López Neyra" (IPBLN-CSIC), Armilla, Granada 18016, Spain. ${ }^{5}$ Biotech Research and Innovation Centre, University of Copenhagen, Ole Maaløes Vej 5, Copenhagen DK-2200, Denmark. ${ }^{6}$ Department of Medicine, University of Cambridge, Hills Road, Cambridge CB2 2QQ, United Kingdom. ${ }^{7}$ The Hamner Institutes for Health Sciences, 6 Davis Drive, Research Triangle Park, Durham, NC 27709, USA.

Received: 8 April 2013 Accepted: 18 October 2013

Published: 28 October 2013

\section{References}

1. Sikorski TW, Buratowski S: The basal initiation machinery: beyond the general transcription factors. Curr Opin Cell Biol 2009, 21:344-351.

2. Rougvie AE, Lis JT: The RNA polymerase II molecule at the $5^{\prime}$ end of the uninduced hsp70 gene of D. melanogaster is transcriptionally engaged. Cell 1988, 54:795-804.

3. Bender TP, Thompson CB, Kuehl WM: Differential expression of c-myb mRNA in murine $B$ lymphomas by a block to transcription elongation. Science 1987, 237:1473-1476.

4. McGeady ML, Wood TG, Maizel JV, Vande Woude GF: Sequences upstream from the mouse c-mos oncogene may function as a transcription termination signal. DNA 1986, 5:289-298.

5. Krumm A, Meulia T, Brunvand M, Groudine M: The block to transcriptional elongation within the human c-myc gene is determined in the promoter-proximal region. Genes Dev 1992, 6:2201-2213.

6. Pinaud S, Mirkovitch J: Regulation of c-fos expression by RNA polymerase elongation competence. J Mol Biol 1998, 280:785-798.

7. Chinsky JM, Maa MC, Ramamurthy V, Kellems RE: Adenosine deaminase gene expression. Tissue-dependent regulation of transcriptional elongation. J Biol Chem 1989, 264:14561-14565.

8. Biragyn A, Nedospasov SA: Lipopolysaccharide-induced expression of TNF-alpha gene in the macrophage cell line ANA-1 is regulated at the level of transcription processivity. J Immunol 1995, 155:674-683. 
9. Raschke EE, Albert T, Eick D: Transcriptional regulation of the lg kappa gene by promoter-proximal pausing of RNA polymerase II. J Immunol 1999, 163:4375-4382.

10. Guenther MG, Levine SS, Boyer LA, Jaenisch R, Young RA: A chromatin landmark and transcription initiation at most promoters in human cells. Cell 2007, 130:77-88.

11. Muse GW, Gilchrist DA, Nechaev S, Shah R, Parker JS, Grissom SF, Zeitlinger J, Adelman K: RNA polymerase is poised for activation across the genome. Nat Genet 2007, 39:1507-1511.

12. Zeitlinger J, Stark A, Kellis M, Hong JW, Nechaev S, Adelman K, Levine M, Young RA: RNA polymerase stalling at developmental control genes in the Drosophila melanogaster embryo. Nat Genet 2007, 39:1512-1516.

13. Core $L$, Lis JT: Transcription regulation through promoter-proximal pausing of RNA polymerase II. Science 2008, 319:1791-1792.

14. Nechaev S, Fargo DC, dos Santos G, Liu L, Gao Y, Adelman K: Global analysis of short RNAs reveals widespread promoter-proximal stalling and arrest of Pol II in Drosophila. Science 2010, 327:335-338.

15. Gilchrist DA, Dos Santos G, Fargo DC, Xie B, Gao Y, Li L, Adelman K: Pausing of RNA polymerase II disrupts DNA-specified nucleosome organization to enable precise gene regulation. Cell 2010, 143:540-551.

16. Levine M: Paused RNA Polymerase II as a Developmental Checkpoint. Cell 2011, 145:502-511.

17. Alexander RD, Innocente SA, Barrass JD, Beggs JD: Splicing-dependent RNA polymerase pausing in yeast. Mol Cell 2010, 40:582-593.

18. Carrillo Oesterreich F, Preibisch S, Neugebauer KM: Global analysis of nascent RNA reveals transcriptional pausing in terminal exons. Mol Cell 2010, 40:571-581.

19. Buratowski S: Progression through the RNA polymerase II CTD cycle. Mol Cell 2009, 36:541-546.

20. Egloff S, Dienstbier M, Murphy S: Updating the RNA polymerase CTD code: adding gene-specific layers. Trends Genet 2012, 28:333-341

21. Bartkowiak B, Mackellar AL, Greenleaf AL: Updating the CTD Story: From Tail to Epic. Genet Res Int 2011, 2011:623718.

22. Hakre S, Chavez L, Shirakawa K, Verdin E: Epigenetic regulation of HIV latency. Curr Opin HIV AIDS 2011, 6:19-24.

23. Wagschal A, Rousset E, Basavarajaiah P, Contreras X, Harwig A, LaurentChabalier S, Nakamura M, Chen X, Zhang K, Meziane O, et al: Microprocessor, Setx, Xrn2, and Rrp6 co-operate to induce premature termination of transcription by RNAPII. Cell 2012, 150:1147-1157.

24. Jones KA, Peterlin BM: Control of RNA initiation and elongation at the HIV-1 promoter. Annu Rev Biochem 1994, 63:717-743.

25. Alcami J, Lain De Lera T, Folgueira L, Pedraza MA, Jacque JM, Bachelerie F, Noriega AR, Hay RT, Harrich D, Gaynor RB, et al: Absolute dependence on kappa $B$ responsive elements for initiation and Tat-mediated amplification of HIV transcription in blood CD4 T lymphocytes. Embo J 1995, 14:1552-1560.

26. Berkhout $B$, Silverman $\mathrm{RH}$, Jeang $\mathrm{KT}$ : Tat trans-activates the human immunodeficiency virus through a nascent RNA target. Cell 1989, 59:273-282.

27. Mancebo HS, Lee G, Flygare J, Tomassini J, Luu P, Zhu Y, Peng J, Blau C, Hazuda D, Price D, Flores O: P-TEFb kinase is required for HIV Tat transcriptional activation in vivo and in vitro. Genes Dev 1997, 11:2633-2644.

28. Wei $\mathrm{P}$, Garber ME, Fang SM, Fischer WH, Jones KA: A novel CDK9associated C-type cyclin interacts directly with HIV-1 Tat and mediates its high-affinity, loop-specific binding to TAR RNA. Cell 1998, 92:451-462.

29. Zhu Y, Pe'ery T, Peng J, Ramanathan Y, Marshall N, Marshall T, Amendt B, Mathews MB, Price DH: Transcription elongation factor P-TEFb is required for HIV-1 tat transactivation in vitro. Genes Dev 1997, 11:2622-2632.

30. Berkhout B, Jeang KT: Functional roles for the TATA promoter and enhancers in basal and Tat-induced expression of the human immunodeficiency virus type 1 long terminal repeat. J Virol 1992, 66:139-149.

31. Lu X, Welsh TM, Peterlin BM: The human immunodeficiency virus type 1 long terminal repeat specifies two different transcription complexes, only one of which is regulated by Tat. J Virol 1993, 67:1752-1760.

32. Olsen HS, Rosen CA: Contribution of the TATA motif to Tat-mediated transcriptional activation of human immunodeficiency virus gene expression. J Virol 1992, 66:5594-5597.

33. Ou SH, Garcia-Martinez LF, Paulssen EJ, Gaynor RB: Role of flanking E box motifs in human immunodeficiency virus type 1 TATA element function. J Virol 1994, 68:7188-7199.

34. Suñé C, Garcia-Blanco MA: Sp1 transcription factor is required for in vitro basal and Tat-activated transcription from the human immunodeficiency virus type 1 long terminal repeat. J Virol 1995, 69:6572-6576.
35. Montanuy I, Torremocha R, Hernandez-Munain C, Suñé C: Promoter influences transcription elongation: TATA-box element mediates the assembly of processive transcription complexes responsive to cyclindependent kinase 9. J Biol Chem 2008, 283:7368-7378.

36. Kato $H$, Sumimoto $H$, Pognonec $P$, Chen $C H$, Rosen CA, Roeder RG: HIVTat acts as a processivity factor in vitro in conjunction with cellular elongation factors. Genes Dev 1992, 6:655-666.

37. Kashanchi F, Piras G, Radonovich MF, Duvall JF, Fattaey A, Chiang CM, Roeder RG, Brady JN: Direct interaction of human TFIID with the HIV-1 transactivator tat. Nature 1994, 367:295-299.

38. Suñé C, Garcia-Blanco MA: Transcriptional trans activation by human immunodeficiency virus type 1 Tat requires specific coactivators that are not basal factors. J Virol 1995, 69:3098-3107.

39. Veschambre $P$, Roisin A, Jalinot $P$ : Biochemical and functional interaction of the human immunodeficiency virus type 1 Tat transactivator with the general transcription factor TFIIB. J Gen Virol 1997, 78(Pt 9):2235-2245.

40. Parada CA, Roeder RG: Enhanced processivity of RNA polymerase II triggered by Tat-induced phosphorylation of its carboxy-terminal domain. Nature 1996, 384:375-378.

41. Wu-Baer F, Sigman D, Gaynor RB: Specific binding of RNA polymerase II to the human immunodeficiency virus trans-activating region RNA is regulated by cellular cofactors and Tat. Proc Natl Acad Sci U S A 1995, 92:7153-7157.

42. Raha T, Cheng SW, Green MR: HIV-1 Tat stimulates transcription complex assembly through recruitment of TBP in the absence of TAFs. PLOS Biol 2005, 3:e44.

43. Lusic M, Marcello A, Cereseto A, Giacca M: Regulation of HIV-1 gene expression by histone acetylation and factor recruitment at the LTR promoter. EMBO J 2003, 22:6550-6561.

44. Wilhelm E, Doyle MC, Nzaramba I, Magdzinski A, Dumais N, Bell B: CTGC motifs within the HIV core promoter specify Tat-responsive pre-initiation complexes. Retrovirology 2012, 9:62

45. Peterlin $\mathrm{BM}$, Price $\mathrm{DH}$ : Controlling the elongation phase of transcription with P-TEFb. Mol Cell 2006, 23:297-305.

46. Coiras M, Lopez-Huertas MR, Perez-Olmeda M, Alcami J: Understanding HIV-1 latency provides clues for the eradication of long-term reservoirs. Nat Rev Microbiol 2009, 7:798-812.

47. Ott M, Geyer M, Zhou Q: The control of HIV transcription: keeping RNA polymerase II on track. Cell Host Microbe 2011, 10:426-435.

48. Zhou Q, Li T, Price DH: RNA polymerase II elongation control. Annu Rev Biochem 2012, 81:119-143.

49. Suñé C, Hayashi T, Liu Y, Lane WS, Young RA, Garcia-Blanco MA: CA150, a nuclear protein associated with the RNA polymerase II holoenzyme, is involved in Tat-activated human immunodeficiency virus type 1 transcription. Mol Cell Biol 1997, 17:6029-6039.

50. Suñé C, Garcia-Blanco MA: Transcriptional cofactor CA150 regulates RNA polymerase II elongation in a TATA-box-dependent manner. Mol Cell Biol 1999, 19:4719-4728.

51. Sanchez-Alvarez M, Goldstrohm AC, Garcia-Blanco MA, Suñé C: Human transcription elongation factor CA150 localizes to splicing factor-rich nuclear speckles and assembles transcription and splicing components into complexes through its amino and carboxyl regions. Mol Cell Biol 2006, 26:4998-5014.

52. Carty SM, Goldstrohm AC, Suñé C, Garcia-Blanco MA, Greenleaf AL: Protein-interaction modules that organize nuclear function: FF domains of CA150 bind the phosphoCTD of RNA polymerase II. Proc Natl Acad SCi USA 2000, 97:9015-9020.

53. Montes M, Cloutier A, Sanchez-Hernandez N, Michelle L, Lemieux B, Blanchette $M$, Hernandez-Munain C, Chabot B, Suñé C: TCERG1 regulates alternative splicing of $\mathrm{BCl}-x$ gene by modulating the rate of RNAPII transcription. Mol Cell Biol 2012, 32:751-762.

54. Singh J, Padgett RA: Rates of in situ transcription and splicing in large human genes. Nat Struct Mol Biol 2009, 16:1128-1133.

55. Lin KT, Lu RM, Tarn WY: The WW domain-containing proteins interact with the early spliceosome and participate in pre-mRNA splicing in vivo. Mol Cell Biol 2004, 24:9176-9185.

56. Smith MJ, Kulkarni S, Pawson T: FF domains of CA150 bind transcription and splicing factors through multiple weak interactions. Mol Cell Biol 2004, 24:9274-9285.

57. Buratowski S: The CTD code. Nat Struct Biol 2003, 10:679-680.

58. Egloff S, Murphy S: Cracking the RNA polymerase II CTD code. Trends Genet 2008, 24:280-288. 
59. Fiscus SA, Pilcher CD, Miller WC, Powers KA, Hoffman IF, Price M, Chilongozi DA, Mapanje C, Krysiak R, Gama S, et al: Rapid, real-time detection of acute HIV infection in patients in Africa. J Infect Dis 2007, 195:416-424.

60. Garcia-Perez J, Sanchez-Palomino S, Perez-Olmeda M, Fernandez B, Alcami J: A new strategy based on recombinant viruses as a tool for assessing drug susceptibility of human immunodeficiency virus type 1. J Med Virol 2007, 79:127-137.

61. Zhou Q, Chen D, Pierstorff E, Luo K: Transcription elongation factor P-TEFb mediates Tat activation of HIV-1 transcription at multiple stages. Embo J 1998, 17:3681-3691.

62. Foskett SM, Ghose R, Tang DN, Lewis DE, Rice AP: Antiapoptotic function of Cdk9 (TAK/P-TEFb) in U937 promonocytic cells. J Virol 2001, 75:1220-1228.

63. Majello B, Napolitano G, Giordano A, Lania L: Transcriptional regulation by targeted recruitment of cyclin-dependent CDK9 kinase in vivo. Oncogene 1999, 18:4598-4605.

64. Pearson JL, Robinson TJ, Munoz MJ, Kornblihtt AR, Garcia-Blanco MA: Identification of the cellular targets of the transcription factor TCERG1 reveals a prevalent role in mRNA processing. J Biol Chem 2008, 283:7949-7961.

65. Ghazi A, Henis-Korenblit S, Kenyon C: A transcription elongation factor that links signals from the reproductive system to lifespan extension in Caenorhabditis elegans. PLOS Genet 2009, 5:e1000639.

66. Miller HB, Saunders KO, Tomaras GD, Garcia-Blanco MA: Tat-SF1 is not required for Tat transactivation but does regulate the relative levels of unspliced and spliced HIV-1 RNAs. PLoS One 2009, 4:e5710.

67. Jablonski JA, Amelio AL, Giacca M, Caputi M: The transcriptional transactivator Tat selectively regulates viral splicing. Nucleic Acids Res 2010, 38:1249-1260.

68. He N, Liu M, Hsu J, Xue Y, Chou S, Burlingame A, Krogan NJ, Alber T, Zhou Q: HIV-1 Tat and host AFF4 recruit two transcription elongation factors into a bifunctional complex for coordinated activation of HIV-1 transcription. Mol Cell 2010, 38:428-438.

69. Sobhian B, Laguette N, Yatim A, Nakamura M, Levy Y, Kiernan R, Benkirane M: HIV-1 Tat assembles a multifunctional transcription elongation complex and stably associates with the 7SK snRNP. Mol Cell 2010, 38:439-451.

70. Shilatifard A, Lane WS, Jackson KW, Conaway RC, Conaway JW: An RNA polymerase II elongation factor encoded by the human ELL gene. Science 1996, 271:1873-1876.

71. Shilatifard A, Duan DR, Haque D, Florence C, Schubach WH, Conaway JW, Conaway RC: ELL2, a new member of an ELL family of RNA polymerase II elongation factors. Proc Natl Acad Sci USA 1997, 94:3639-3643.

72. Nagel JE, Smith RJ, Shaw L, Bertak D, Dixit VD, Schaffer EM, Taub DD Identification of genes differentially expressed in T cells following stimulation with the chemokines CXCL12 and CXCL10. BMC Immunol 2004, 5:17.

73. Brass AL, Dykxhoorn DM, Benita Y, Yan N, Engelman A, Xavier RJ, Lieberman J, Elledge SJ: Identification of host proteins required for HIV infection through a functional genomic screen. Science 2008, 319:921-926.

74. Konig R, Zhou Y, Elleder D, Diamond TL, Bonamy GM, Irelan JT, Chiang CY, Tu BP, De Jesus PD, Lilley CE, et al: Global analysis of host-pathogen interactions that regulate early-stage HIV-1 replication. Cell 2008, 135:49-60.

75. Zhou H, Xu M, Huang Q, Gates AT, Zhang XD, Castle JC, Stec E, Ferrer M, Strulovici B, Hazuda DJ, Espeseth AS: Genome-scale RNAi screen for host factors required for HIV replication. Cell Host Microbe 2008, 4:495-504.

76. Murali TM, Dyer MD, Badger D, Tyler BM, Katze MG: Network-based prediction and analysis of HIV dependency factors. PLoS Comput Biol 2011, 7:e1002164.

77. Adachi A, Gendelman HE, Koenig S, Folks T, Willey R, Rabson A, Martin MA: Production of acquired immunodeficiency syndrome-associated retrovirus in human and nonhuman cells transfected with an infectious molecular clone. J Virol 1986, 59:284-291.

78. Alvarez-Fernandez C, Crespo Guardo A, Garcia-Perez J, Garcia F, Blanco J, Escriba-Garcia L, Gatell JM, Alcami J, Plana M, Sanchez-Palomino S: Generation and Characterization of a Defective HIV-1 Virus as an Immunogen for a Therapeutic Vaccine. PLoS One 2012, 7:e48848.

79. Bachelerie F, Alcami J, Arenzana-Seisdedos F, Virelizier JL: HIV enhancer activity perpetuated by NF-kappa B induction on infection of monocytes. Nature 1991, 350:709-712.

80. Arenzana-Seisdedos F, Fernandez B, Dominguez I, Jacque JM, Thomas D, Diaz-Meco MT, Moscat J, Virelizier JL: Phosphatidylcholine hydrolysis activates NF-kappa B and increases human immunodeficiency virus replication in human monocytes and T lymphocytes. J Virol 1993, 67:6596-6604.

81. Bradford MM: A rapid and sensitive method for the quantitation of microgram quantities of protein utilizing the principle of protein-dye binding. Anal Biochem 1976, 72:248-254.

82. Jablonski JA, Caputi M: Role of cellular RNA processing factors in human immunodeficiency virus type 1 mRNA metabolism, replication, and infectivity. J Virol 2009, 83:981-992.

83. Lain De Lera T, Folgueira L, Martin AG, Dargemont C, Pedraza MA, Bermejo $\mathrm{M}$, Bonay $\mathrm{P}$, Fresno $\mathrm{M}$, Alcami J: Expression of IkappaBalpha in the nucleus of human peripheral blood T lymphocytes. Oncogene 1999, 18:1581-1588.

doi:10.1186/1742-4690-10-124

Cite this article as: Coiras et al:: Transcription elongation regulator 1 (TCERG1) regulates competent RNA polymerase II-mediated elongation of HIV-1 transcription and facilitates efficient viral replication. Retrovirology 2013 10:124

\section{Submit your next manuscript to BioMed Central and take full advantage of:}

- Convenient online submission

- Thorough peer review

- No space constraints or color figure charges

- Immediate publication on acceptance

- Inclusion in PubMed, CAS, Scopus and Google Scholar

- Research which is freely available for redistribution
C) Biomed Central 